\title{
Two Different Approaches for Consistency of Intuitionistic Multiplicative Preference Relation using Directed Graph
}

Mamata Sahu

Delhi Technological University

Anjana Gupta ( $\square$ anjanagupta@dce.ac.in )

Delhi Technological University

\section{Research Article}

Keywords: Isomorphism, consistency, Directed graph, cycle, Intuitionistic multiplicative preference relation, asymmetric multiplicative preference relation

Posted Date: September 7th, 2021

DOI: https://doi.org/10.21203/rs.3.rs-847781/v1

License: (c) (i) This work is licensed under a Creative Commons Attribution 4.0 International License.

Read Full License

Version of Record: A version of this preprint was published at Soft Computing on March 28th, 2022. See the published version at https://doi.org/10.1007/s00500-022-06965-5. 


\title{
Two different Approaches for consistency of Intuitionistic Multiplicative Preference Relation using Directed Graph
}

\author{
Mamata Sahu ${ }^{1} \cdot$ Anjana Gupta ${ }^{2, *}$
}

Received: date / Accepted: date

\begin{abstract}
Consistency is an important issue that causes wide public concern of decision-makers in the decision-making process. The lack of consistency in preference relations results in a vague solution. The main goal of this paper is to achieve the consistent intuitionistic multiplicative preference relation using a graphical approach. We have proposed two different characterizations of the consistency for intuitionistic multiplicative preference relation(IMPR). In the first approaches, we propose an algorithm to achieve the consistency of IMPR by using the cycles of various length in a directed graph. The second approach proves isomorphism between the set of IMPRs and the set of asymmetric multiplicative preference relations. That result is explored to use the methodologies developed for asymmetric multiplicative preference relations to the case of IMPRs and achieve the consistency of asymmetric multiplicative preference relation using a directed graph. Sometimes the decision maker may not be able to provide the complete relation. So the above-said method is applied for an incomplete IMPR also, here consistency plays an important role. The examples are provided to illustrate both the methods in all cases.
\end{abstract}

Keywords Isomorphism, consistency, Directed graph, cycle, Intuitionistic multiplicative preference relation, asymmetric multiplicative preference relation.

\section{Introduction}

In decision-making problems, preference relation is one of the most useful tools used by decision makers(DM's), as it can express their views over the alternatives/criteria. In the past decades, plenty of research has been done about preference relations. Among these efforts, the fuzzy preference relations (Orlovsky, 1978) and the multiplicative preference relations (Saaty, 1980) are two types of preference relations which are widely studied by many researchers. Additionally, the multiplicative preference relations have been deeply studied and successfully applied in the analytic hierarchy process (AHP)(Saaty, 1980). In fuzzy preference relation(FPR), expert provides the preference information using the 0-1 scale, and a multiplicative preference relation (MPR) (Saaty, 1980) in which the (1/9)-9 ratio scale is used to measure the intensity of the pairwise comparison of alternatives.

In these preference relations, the essential elements provide the degree that an alternative is prior to the other. However, sometimes DM's may also need to give the degree that an alternative is not prior to the other in some practical problems. In these cases, DM not sure about the preference information namely, hesitation degree (uncertainty degree). Intuitionistic fuzzy preference relations (IFPRs) (Xu 2007b) and intuitionistic multiplicative preference relations (IMPRs) (Xia et al., 2013) are proposed to indicate the

\footnotetext{
${ }^{1}$ Department of Applied Mathematics, Delhi Technological University, Delhi 110042, India E-mail: munmun.sahu@gmail.com .

2,* Corresponding author, Department of Applied Mathematics, Delhi Technological University, Delhi 110042, India E-mail: anjanagupta@dce.ac.in
} 
positive information $x_{i}$ is preferred to $x_{j}$, the degree of negative details $x_{i}$ is not preferred to $x_{j}$ and simultaneously the degree that cannot determine by the DM.

The consistency, a fundamental property of preference relations, in the decision-making process has drawn much attention in many decision-making fields. The level of agreement among the preference data given by the individual DMs' measures the consistency (Xia, Xu and Chen 2013). Based on the multiplicative consistency property, Xia et al.(Xia, Xu and Chen 2013) discussed the consistency of FPRs and developed an algorithm to improve the consistency level of FPRs. Herrera-Viedma et al. (2004) developed a method for constructing consistent FPRs and using the additive transitivity property of the FPRs they proposed a characterization of the consistency property. Chiclana et al. (2009) put forwarded a functional equation to demonstrate the "cardinal consistency in the strength of preferences" of reciprocal preference relations (RPRs).

Different type of reciprocal preference relations surveyed by Li et al. (2019) using the additive consistency. Furthermore, they research some fusion methods and consistency-driven decision making methods.

Capuano et al. (2018) proposed a new influence-guided GDM model and also the effects of social influence are generally relevant to cases when the presences of expertŠs knowledge are not known. Then use social influence network (SIN) to calculate the missing preferences and develop them by simulating the effect of the expert's interpersonal influence before aggregating them to select the best alternative. Ureña et al.(2015) reviewed and analyzed the state-of-the-art research efforts on group decision making from the perspective of the estimation of missing preferences using different types of preference relations. These estimation techniques mainly use the properties of additive or multiplicative consistency for the missing options among the known options, as well as to increase the overall consistency. In many cases it is the consensus of experts.

To improve the consistency of a MPR Xu and Wei (1999) proposed an algorithm to derive a definite reciprocal matrix with acceptable consistency. Ergu et al. (2011) developed a method to measure the consistency for MPRs.

$\mathrm{Xu}$ et al. (2013) first proposed the ordinal consistency of a FPR, then the authors proposed how to find the cycles in the direct graphs. In addition, they also proposed the process of calculating the ordinal consistency index and positioning each cycle, as well as the process of finding inconsistent judgments in the preference relationship. To repair the inconsistency in FPR, they developed an algorithm to find and eliminate the 3 cycles on the graph. Xu, Ma and Herrera (2019) first proposed the ordinal consistency of incomplete fuzzy linguistic preference relations. Also, they developed one novel algorithm to judge whether an incomplete FLPR is ordinaly consistent. To rectify the inconsistency of an incomplete FLPR they developed an another algorithm and improve it for ordinally consistent. Using the proposed algorithms they identify and rectify the ordinal inconsistency of the complete FLPRs. Xu et al. (2018) studied both the ordinal and multiplicative consistencies for FPRs. To measure the degree of ordinal consistency for FPRs, they introduced a new ordinal consistency index, and developed a new multiplicative inconsistency identification and modification method for FPR. Xu et al. (2021) developed an algorithms to rectify the multiplicative and ordinal inconsistencies for FPRs. Xu and Herrera (2019) proposed a graphical method to visualize and rectify different inconsistencies for FPRs. Using, ordinal and cardinal consistency, on a two-dimensional plan, they draw Gower plots to visualize the ordinal and cardinal inconsistencies and they formulated some optimization models to rectify different types of inconsistences, respectively. Xu, Herrera and Wang (2016) proposed a distance-based methodology is proposed to deal with ordinal and additive inconsistencies for FPR.

A large number of researchers are committed to using IFPR to make decisions in uncertain environments (Xu and Liao, 2015). The definitions of feasible-region-based consistency and priority derivation methods were proposed for IFPRs(Behret 2014, Gong, et al., 2011, Gong et al. 2009).

Jiang et al. (2015) discussed the consistency and acceptably consistent of an IMPR. Based on it, two approaches were developed to complement all missing elements of incomplete IMPRs. Ren et al. 
(2016) defined IMWGA operator and proposed an iterative process to adjust the inconsistent IMPRs into an acceptably consistent one and repair the maximum in conformity gradually, which makes fewer changes to the DMs' original opinions. Also, they provide an adjustment process to restore and improves the consistency of inconsistent IMPR. Zhang and Guo (2017) developed a linear programming-based algorithm to check and adjust the flexibility of an IMPR. As well, Zhang and Guo (2017) discuss the relationships between an IMPR and a normalized intuitionistic multiplicative weight vector and developed two group decision methods based on complete and incomplete IMPRs, respectively. Zhang and Pedrycz (2018) manage the unity and consensus of IMPR, and establish a consistent and consensus-based method through various proposed goal programming models to address group decision making (GDM) with IMPR. In this paper, our work focuses on only IMPRs.

\subsection{Focus of the present study}

In 1995, Nishizawa (Nishizawa 1995) proposed an algorithm checking for the consistency of MPRs by using the cycle of a directed graph. After that Nishizawa (1996), proposed two algorithms to find the various cycles of odd and even length in the incomplete directed graph. Taking inspiration from the Nishizawa (1996), in this paper, we have developed two approaches to improve the consistency of IMPR. In the first approach, we have formed an ordered-pair binary matrix from the IMPR and which is split into two binary arrays and using Nishizawa (1996) algorithm, we have checked the consistency of IMPR.

The subsequent methodology, of this paper, is to demonstrate the isomorphism between the set of IMPR and the set of asymmetric multiplicative preference relations. This outcome can accordingly be abused to utilize techniques created for MPRs to the instance of IMPR and, eventually, to expand the utilization of IMPR in decision making and to overcome the computation of above mentioned complexity. In other words, this result will allow taking advantage of mature and well-defined methodologies developed for MPRs while controlling the flexibility of IMPR to model vagueness/uncertainty.

As shown in work mentioned above, the preference relations are generally with complete information. In the decision-making process, DM required $\frac{n(n-1)}{2}$ judgement at each level to present an entire preference relation, and when $n$ is large, it becomes a tedious task. Here and there, DM may not yet have a good understanding of a particular question, and thus he/she is unable to make a direct comparison between every two objects. Therefore it is sometimes necessary to allow the DM to skip some dubious comparisons flexibly. Thus, due to lack of time and busy schedule of the DM sometimes incomplete preference relations are obtained. In this case, the whole process may slow down. Therefore in this paper, both the approaches are applied in incomplete IMPR scenario.

Indeed, an issue that can be addressed using the mentioned equivalence is the presence of incomplete IMPR in the decision-making process. Using directed graph (Nishizawa, 1996) we check the consistency of asymmetric multiplicative preference relation. A new section has been added that compare the two approaches and improve the consistency of IMPR.

\subsection{Organization of the paper}

The rest of the paper is arranged as follows. Section 2 represent some basic concepts of MPRs and IMPRs. We have developed two approaches to check the consistency of IMPR given in section 3 and 4 . Section 3 demonstrates the first approach in which, we have developed an algorithm that improves the consistency of IMPRs and is discussed with some examples. This section includes incomplete IMPRs also. The second approach in section 4, the set of IMPR and the set of asymmetric multiplicative preference relations has proved mathematically isomorphic. Numerical examples are also given in this section for both complete or incomplete intuitionistic scenario. In this section, we have given a comparison between 
the two approaches and improve the consistency of IMPR. Section 5 gives a comparative analysis of the proposed methodology relevant to the context. Concluding remarks are given in the last section.

\section{Preliminaries}

Before introducing the basic concept of MPR and IMPR, first we have discussed the analytical hierarchy process.

Generally, in the decision-making problems, Analytical hierarchy process (AHP) is applied with a finite number of alternatives. There are limited arrangement choices $X=\left\{x_{1}, \cdots, x_{n}\right\}$ and there is one objective in the decision process, from which the DM, is typically approached to choose the best one. Generally, in the decision-making process, DM is closest to assigning a score to each of the other options and then selecting the option with the most extreme value. That is, for a given arrangement of choices, $X=\left\{x_{1}, \cdots, x_{n}\right\}$, the DM ought to give a weight vector of

$$
w=\left(w_{1}, \cdots, w_{n}\right)^{T}
$$

where, $w_{i}$ is a worth that correctly determine the score of option $x_{i}$. The weight vector $w_{i}$ is more prominent, that better the $i$-th alternative. As demonstrated by the theory of Fishburn (Fishburn 1979), the rule is that if and only if $w_{i}>w_{j}$, the alternative $x_{i}$ is preferred to the alternative $x_{j}$. The components $w_{i}$ of the weight vectors are called priorities, or weights, of the alternatives $x_{i}$, and on the other hand weight vectors are used for ranking of alternatives. In particular, $w=(0.1,0.4,0.2,0.3)^{T}$ infers $x_{2} \succ x_{4} \succ x_{3} \succ x_{1}$ where $x_{i} \succ x_{j}$ implies that option $x_{i}$ is preferred to $x_{j}$. Along these lines, the decisions process appears to be simple, but when complexity increases, it turns into a hard errand. As we will see, complexity increases affectionately intertwined with the quantity of choices furthermore, criteria. A DM could continue running into bothers when mentioned to exhibit a rating as a numerical vector for an enormous number of options. Some times, it happens that we can not settle on a few other options. Far and away more terrible, we can not choose what's more, in the end, understand that it was not the best choice. There is an effective way to use pairwise comparisons to overcome this problem. The explanation for this is that it allows DMs to consider two options at the same time. Afterwards, the technique is to decompose the original problem into many smaller sub-problems and manage the latter. Formally, from the set of pairwise comparisons, the pairwise comparison matrix $A=\left(a_{i j}\right)_{n \times n}$, can be structured as follows

$$
A=\left(\begin{array}{cccc}
a_{11} & a_{12} & \cdots & a_{1 n} \\
a_{21} & a_{22} & \cdots & a_{2 n} \\
\vdots & \vdots & \ddots & \vdots \\
a_{n 1} & a_{n 2} & \cdots & a_{n n}
\end{array}\right)
$$

with $a_{i j}>0$ communicating the level of inclination of $x_{i}$ to $x_{j}$. More precisely, according to Saaty's theory, each entry must approximate the ratio between the two weights

$$
a_{i j} \approx \frac{w_{i}}{w_{j}}
$$

This implies, on the off chance that the entries precisely speak to proportions between weights, at that point the comparison matrix $A$ can be communicated in the accompanying structure,

$$
A=\left(\frac{w_{i}}{w_{j}}\right)_{n \times n}=\left(\begin{array}{cccc}
\frac{w_{1}}{w_{1}} & \frac{w_{1}}{w_{2}} & \cdots & \frac{w_{1}}{w_{n}} \\
\frac{w_{2}}{w_{1}} & \frac{w_{2}}{w_{2}} & \cdots & \frac{w_{2}}{w_{n}} \\
\vdots & \vdots & \ddots & \vdots \\
\frac{w_{n}}{w_{1}} & \frac{w_{n}}{w_{2}} & \cdots & \frac{w_{n}}{w_{n}}
\end{array}\right)
$$


Table 1 Random Index

\begin{tabular}{|c|c|c|c|c|c|c|c|c|c|c|}
\hline $\mathrm{n}$ & 1 & 2 & 3 & 4 & 5 & 6 & 7 & 8 & 9 & 10 \\
\hline R.I & 0 & 0 & 0.52 & 0.89 & 1.11 & 1.25 & 1.35 & 1.40 & 1.45 & 1.49 \\
\hline
\end{tabular}

Note that, when we represent 1 and consider the 2 , one condition of multiplicative reciprocity $a_{i j}=$ $\frac{1}{a_{j i}} \forall i, j$ holds. The matrix $A$ can be improved and rewritten as,

$$
A=\left(\begin{array}{cccc}
1 & a_{12} & \cdots & a_{1 n} \\
\frac{1}{a_{12}} & 1 & \cdots & a_{2 n} \\
\vdots & \vdots & \ddots & \vdots \\
\frac{1}{a_{1 n}} & \frac{1}{a_{2 n}} & \cdots & 1
\end{array}\right)
$$

In other words, the rearrangement structure of this form of pairwise comparison matrix comes from assumptions. Namely, when the option $x_{1}$ is twice as good as $x_{2}$, then we can infer that $x_{2}$ is $1 / 2$ of $x_{1}$. We are currently continuing this example, and imagine that the pairwise comparison matrix of a city has $X$ set to features and $x_{1}, x_{2}, x_{3}$ are appended to the rows and columns of the matrix.

$$
A=\left(\begin{array}{ccc}
1 & 3 & 6 \\
1 / 3 & 1 & 2 \\
1 / 6 & 1 / 2 & 1
\end{array}\right)
$$

In particular, the element $a_{12}$ of this matrix $A$ says that the alternative $x_{1}$ is considered 3 times better than $x_{2}$, i.e. $a_{12}=3$.

There are many ways to find the priority vector $w$ from the pairwise comparison matrix. In this example, it can be checked that the condition $a_{i j}=w_{i} / w_{j}, \forall i, j$ is satisfied, for instance, the sum of the following vector and its components is 1 i.e. $w=(6 / 9,2 / 9,1 / 9)^{T}$.

Several basic concepts related to MPR and IMPR are introduced, and accordingly the concept of incomplete IMPR is defined.

Definition 1 (Saaty, 1980) MPR $R=\left(r_{i j}\right)_{n \times n}$ is called multiplicative consistent if it satisfied the multiplicative transitivity property

$$
r_{i j}=r_{i k} r_{k j}, \forall i, j, k=1,2, \cdots, n
$$

In this case, there exit a positive vector $w=\left(w_{1}, w_{2}, \cdots, w_{n}\right)^{T}$ such that $r_{i j}=\frac{w_{i}}{w_{j}}$ satisfying the conditions $\sum_{i=1}^{n} w_{i}=1, w_{i} \geq 0, i, j \in N$, where $w_{i}$ is the priority weight.

In general cases, equation 3 does not always hold, therefore the MPRs are inconsistent. Saaty (1980) gave a method to measure the consistency degree of an MPR for an inconsistent MPR $R$, by the concept of consistency index (CI) and consistency ratio (CR).

$$
C I=\frac{\lambda_{\max }-n}{n-1}, C R=\frac{C I}{R I_{n}}
$$

where $\lambda_{\max }$ and $n$ are the largest eigen value and the order of the MPR $R$ respectively. $R I_{n}$ is the random index that dependents on the orders of the preference relation matrices given in the table 1 .

Definition 2 An MPR $R$ is said to be acceptably consistent if $C R \leq 0.1$, else $R$ is announced not acceptably consistent (or simply inconsistent), and returned to an expert to have a re-look at the preferences evaluations. 
Expressly, if the value of the consistency ratio $(C R)<0.1$, the comparison matrix is acceptable. There is no standard for matrix consistency. According to some experiments and experiences, the degree of inconsistency is $10 \%$ of the degree of inconsistency still acceptable.

Nishizawa, in 1995 proposed an algorithm for the consistency of MPRs by using the cycle of a directed graph. Later Nishizawa (1996) proposed two algorithms to find the cycles of various odd and even length using incomplete directed graph. For finding cycles, vertex matrix that is denoted by $V$ of order $n \times n$ is needed, among them, $n$ is the number of vertices, corresponding to the element $(i, j)$, which is a directed graph determined by $V(i, j)$. If one points $i$ is connected to another point $j$ by an arrow, say " $i \rightarrow j$ ", then $V(i, j)=1$ otherwise 0 . Pairwise comparison data is represented by $\theta$ or $1 / \theta$, where $\theta$ is the parameter whose binary AHP value is greater than 1 (Saaty, 1977). Nishizawa proposed two algorithms (Nishizawa, 1996) for even and odd length cycle in the incomplete directed graph. From the result Nishizawa (1996) one can easily judge the consistency of comparison matrix. For the sake of convenience, following the two algorithms are given by Nishizawa (1996) for finding the cycle of odd and even length.

Algorithm 1 (Nishizawa, 1996)(Finding even length cycle) Nishizawa (1996) discussed the cycle of even length $2 m(m=2,3, .$.$) , of n$ elements be the form of $\left(i_{0} i_{1} \cdots i_{m-1} j_{0} j_{1} ; \cdots j_{m-1}\right)$.

1. Find the vertex matrix $V$ and $V^{m}$.

2. Find pair of numbers $i_{0}$ and $j_{0}$ with $V^{m}\left(i_{0}, j_{0}\right)>0$ and $V^{m}\left(j_{0}, i_{0}\right)>0$, where $i_{0}<j_{0}$. Without these pairs, there are no cycles that are $2 m$ in length.

3. Find a pairs of numbers $i_{\alpha}\left(>i_{0}\right)$ and $j_{\alpha}\left(>i_{0}\right)$ under the conditions $V\left(i_{\alpha-1}, i_{\alpha}\right)=1, V^{m}\left(i_{\alpha}, j_{\alpha}\right)>0$, $V^{m}\left(j_{\alpha}, i_{\alpha}\right)>0$, and $V\left(j_{\alpha-1}, j_{\alpha}\right)=1$ for each $\alpha=1 \sim m-1$.

4. Confirm $V\left(i_{m-1}, j_{0}\right)=1$ and $V\left(j_{m-1}, i_{0}\right)=1$.

5. Repeat step 2 to 4 for all pairs of $i_{0}, j_{0}$ where $i_{0}<j_{0}$.

Algorithm 2 (Nishizawa, 1996)(Finding odd length cycle) Nishizawa (1996) discussed the cycle of odd length $2 m-1(m=2,3, .$.$) , of n$ elements be the form of $\left(i_{0} i_{2} \cdots i_{m-3} j_{m-2} \cdots j_{3} j_{1} k i_{1} i_{3} \cdots i_{m-2} j_{m-3}\right.$ $\left.\cdots j_{2} j_{0}\right)$ if $m$ is odd, and $\left(i_{0} i_{2} \cdots i_{m-2} j_{m-3} \cdots j_{3} j_{1} k i_{1} i_{3} \cdots i_{m-3} j_{m-2} \cdots j_{2} j_{0}\right)$ if $m$ is even.

1. Find the vertex matric $V, V^{m-1}$, and $V^{m}$.

2. Find pair of numbers $i_{0}, \mathrm{k}$ and $j_{0}$ with $V^{m-1}\left(i_{0}, k\right)>0$ and $V^{m}\left(k, i_{0}\right)>0, V^{m-1}\left(k, j_{0}\right)>0$, $V^{m}\left(j_{0}, k\right)>0$, and $V\left(j_{0}, i_{0}\right)=1$, where $i_{0}<k, i_{0}<j_{0}$. Without these pairs, there are no cycles of length $2 m-1$.

3. Find pair of numbers $i_{1}\left(>i_{0}\right)$ and $j_{1}\left(>i_{0}\right)$ with $V^{m}\left(i_{0}, i_{1}\right)>0, V^{m-1}\left(i_{1}, i_{0}\right)>0, V\left(k, i_{1}\right)=1$, $V^{m-1}\left(j_{0}, j_{1}\right)>0, V^{m}\left(j_{1}, j_{0}\right)>0$, and $V\left(j_{1}, k\right)=1$.

4. Find a pairs of numbers $i_{\alpha}\left(>i_{0}\right)$ and $j_{\alpha}\left(>i_{0}\right)$ under the conditions $V^{m}\left(i_{\alpha-1}, i_{\alpha}\right)>0, V^{m-1}\left(i_{\alpha}, i_{\alpha-1}\right)>$ $0, V\left(i_{\alpha-2}, i_{\alpha}\right)=1, V^{m-1}\left(j_{\alpha-1}, j_{\alpha}\right)>0, V^{m}\left(j_{\alpha}, j_{\alpha-1}\right)>0$, and $V\left(j_{\alpha}, j_{\alpha-2}\right)=1$ for each $\alpha=2 \sim$ $m-2$.

5. Confirm $V\left(i_{m-2}, j_{m-3}\right)=1$ and $V\left(i_{m-3}, j_{m-2}\right)=1$.

6. Repeat step 2 to 4 for all pairs of $i_{0}, \mathrm{k}, j_{0}$ where $i_{0}<k, i_{0}<j_{0}$.

If no cycles have found in the directed graph, then the comparison matrix is consistent. The comparison matrix is inconsistent if at least one cycle is found in the incomplete directed graph. In case of inconsistency, they find the minimum covering sets (Nishizawa, 1995) among the cycles, and then they eliminate the path of cycles such as the comparison matrix is consistent.

In this paper, we have discussed two approaches for checking the consistency of IMPRs. Here we are extending the above method of Nishizawa (1996) in IMPR scenario. For this, we recall some basic concept of IMPRs, incomplete IMPRs, and the consistency property. Xia et al. (2013) extended MPR into IMPR.

Definition 3 (Xia et al. 2013)Let $\tilde{R}=\left[\tilde{r}_{i j}\left(x_{i}, x_{j}\right)\right]_{n \times n}$ be an intuitionistic multiplicative preference relation (IMPR), where $\tilde{r}_{i j}\left(x_{i}, x_{j}\right)=\left(\wp\left(x_{i}, x_{j}\right), \Im\left(x_{i}, x_{j}\right)\right), i, j \in N$, is an intuitionistic multiplicative 
number (IMN), and $\wp\left(x_{i}, x_{j}\right)$ indicates certainty degree to which $x_{i}$ is preferred to $x_{j}$ and $\Im\left(x_{i}, x_{j}\right)$ is the certainty degree to which $x_{i}$ is not preferred to $x_{j}$, and it satisfy the following characteristics:

$$
\begin{gathered}
1 / 9 \leq \wp\left(x_{i}, x_{j}\right), \quad \Im\left(x_{i}, x_{j}\right) \leq 9, \wp\left(x_{i}, x_{j}\right)=\Im\left(x_{j}, x_{i}\right), \Im\left(x_{i}, x_{j}\right)=\wp\left(x_{j}, x_{i}\right), \\
\wp\left(x_{i}, x_{i}\right)=\Im\left(x_{i}, x_{i}\right)=1, \quad 0<\wp\left(x_{i}, x_{j}\right) \Im\left(x_{i}, x_{j}\right) \leq 1, \quad \forall i, j \in N .
\end{gathered}
$$

If $\wp\left(x_{i}, x_{j}\right) . \Im\left(x_{i}, x_{j}\right)=1, \forall i, j \in N$, the IMPR is equivalent to an MPR. The value of $\wp\left(x_{i}, x_{j}\right), \Im\left(x_{i}, x_{j}\right)$ are not arbitrary. For easy understanding $\wp\left(x_{i}, x_{j}\right)$ and $\Im\left(x_{i}, x_{j}\right)$ are denoted by $\wp_{i j}$ and $\Im_{i j}$ respectively.

$\mathrm{Xu}$ (2013) has given the consistent property of IMPR $\tilde{R}=\left(\tilde{r}_{i j}\right)_{n \times n}=\left(\wp_{i j}, \Im_{i j}\right)_{n \times n}$ based on the transitive property:

$$
\left(\wp_{i j}, \Im_{i j}\right)=\left(\wp_{i k} \wp_{k j}, \Im_{i k} \Im_{k j}\right) \text {, for all } i, j, k \in N \text { and } i \leq k \leq j \text {. }
$$

It is to note that the equation (5) is restricted for the condition $i \leq k \leq j$, while the transitive property of a MPR is unconstrained which satisfies for all $i, j, k \in N$. If the equation (5) is utilized to check the consistency of an IMPR for all $i, j, k \in N$, the transitivity and consistency properties some times do not hold. This is because that when ' $k$ ' comes from the row of the lower triangular matrix, the equation does not hold. For example:

$$
\left(\begin{array}{ccc}
(1,1) & (1 / 2,1) & (1,1 / 2) \\
(1,1 / 2) & (1,1) & (2,1 / 2) \\
(1 / 2,1) & (1 / 2,2) & (1,1)
\end{array}\right)_{3 \times 3}
$$

is a consistent IMPR given by $\mathrm{Xu}, 2013$. Jiang et al. (2015) relax the condition $i \leq k \leq j$, it is follow that $a_{23}=\left(\wp_{21} \wp_{13}, \Im_{21} \Im_{13}\right)=(1,1 / 4)$. But $a_{23}=(2,1 / 2) \neq(1,1 / 4)$. To over come this type of transitivity limitation, Jiang et al. (2015) proposed a more general consistency property of an IMPR split into two MPRs by using the formula

$$
c_{i j}=\left\{\begin{array}{ll}
\wp_{i j} & i<j \\
1 & i=j \\
1 / \Im_{i j} & i>j
\end{array} \text { and } d_{i j}= \begin{cases}\Im_{i j} & i<j \\
1 & i=j \\
1 / \wp_{i j} & i>j .\end{cases}\right.
$$

where the MPRs $C=\left(c_{i j}\right)_{n \times n}$ and $D=\left(d_{i j}\right)_{n \times n}$ are preferred and non-preferred information matrix given by the DM with respect to the alternative $x_{i}$ over $x_{j}$. Based on the above concept, Jiang et al. (2015) defined the consistent IMPR.

Jiang et al. (2015) relax the condition $i \leq k \leq j$ and proposed a more general consistency property of IMPR to overcome this type of transitivity limitation.

Definition 4 (Jiang et al. 2015) An IMPR $\tilde{R}=\left(\tilde{r}_{i j}\right)_{n \times n}$ is said to be consistent if both MPRs $C$ and $D$ obtained by splitting the IMPR $\tilde{R}$ are consistent such that

$$
c_{i j}=c_{i k} c_{k j}, d_{i j}=d_{i k} d_{k j} \forall i, j, k \in N
$$

In this paper we have developed two different approaches to check the consistency of IMPR and incomplete IMPRs which are discussed in section 3 and 4 respectively.

\section{Consistency for IMPR and Incomplete IMPR}

The absence of consistency in decision making with preference relations is a big challenge to bring about conflicting conclusions. Numerous strategies on consistency measure and improvement of preference relations with various structures have been exhibited progressively. This section checks the consistency of both IMPR and incomplete IMPR. 
Let $\tilde{R}$ be the IMPR,

$$
\tilde{R}=\left(\begin{array}{cccccc}
\left(\wp_{11}, \Im_{11}\right) & \left(\wp_{12}, \Im_{12}\right) & \cdots & \left(\wp_{1 i}, \Im_{1 i}\right) & \cdots & \left(\wp_{1 n}, \Im_{1 n}\right) \\
\left(\wp_{21}, \Im_{21}\right) & \left(\wp_{22}, \Im_{22}\right) & \cdots & \left(\wp_{2 i}, \Im_{2 i}\right) & \cdots & \left(\wp_{2 n}, \Im_{2 n}\right) \\
\vdots & \vdots & \ddots & \vdots & \ddots & \vdots \\
\left(\wp_{i 1}, \Im_{i 1}\right) & \left(\wp_{i 2}, \Im_{i 2}\right) & \cdots & \left(\wp_{i i}, \Im_{i i}\right) & \cdots & \left(\wp_{i n}, \Im_{i n}\right) \\
\vdots & \vdots & \ddots & \vdots & \ddots & \vdots \\
\left(\wp_{n 1}, \Im_{n 1}\right) & \left(\wp_{n 2}, \Im_{n 2}\right) & \cdots & \left(\wp_{n i}, \Im_{n i}\right) & \cdots & \left(\wp_{n n}, \Im_{n n}\right)
\end{array}\right)
$$

We define a ordered pair vertices matrix $V=\left\{v_{i j}\right\}=\{(a, b)\}$ as follows, where $v_{i j}$ is an ordered pair $(a, b)$ such that

$$
\left\{\begin{array}{l}
\text { For } i<j \begin{cases}\text { if } \wp_{i j}>1 & \text { then } a=1, \text { otherwise } a=0 \\
\text { if } \Im_{i j}>1 & \text { then } b=1, \text { otherwise } b=0\end{cases} \\
\text { For } i>j \begin{cases}\text { if } \frac{1}{\Im_{i j}}>1 & \text { then } a=1, \text { otherwise } a=0 \\
\text { if } \frac{1}{\wp_{i j}}>1 & \text { then } b=1, \text { otherwise } b=0\end{cases} \\
\text { For } i=j \text { both } a=0 \text { and } b=0 .
\end{array}\right.
$$

To illustrate this consider the following IMPR

$$
\left(\begin{array}{ccc}
(1,1) & (1 / 2,2 / 3) & (1 / 5,5) \\
(2 / 3,1 / 2) & (1,1) & (4 / 5,3 / 4) \\
(5,1 / 5) & (3 / 4,4 / 5) & (1,1)
\end{array}\right)
$$

Using the binary order paired vertex matrix $V$ is given below

$$
V=\left(\begin{array}{ccc}
(0,0) & (0,0) & (0,1) \\
(1,1) & (0,0) & (0,0) \\
(1,0) & (1,1) & (0,0)
\end{array}\right)
$$

Here, the binary order paired vertex matrix is split into two vertex matrix i.e lower vertex matrix $V_{L}$ containing the lower element of order pair and upper vertex matrix $V_{U}$ containing the upper element of order pair.

$$
V_{L}=\left(\begin{array}{lll}
0 & 0 & 0 \\
1 & 0 & 0 \\
1 & 1 & 0
\end{array}\right), \quad V_{U}=\left(\begin{array}{lll}
0 & 0 & 1 \\
1 & 0 & 0 \\
0 & 1 & 0
\end{array}\right)
$$

Using the concept of order pair, we have defined a new definition of consistent IMPR.

Definition 5 The IMPR is consistent if both the corresponding lower and upper vertex matrices are consistent.

In this section, we have applied the graphical approach of Nishizawa (1996) to check the consistency of both complete IMPRs and incomplete IMPRs scenario. We have developed an algorithm to illustrate the above method.

Algorithm 3 Step1: Let us consider an IMPR.

Step2: Order pair vertex matrix is obtained using equation 8. In case of incomplete IMPRs, missing elements are treated as zero in the vertex matrix.

Step3: The ordered pair vertex matrix is split into the lower vertex matrix and upper vertex matrix.

Step4: Then apply the algorithm 1 and 2 of section 2 for finding the cycle of even and odd length on both the vertex matrix.

Step5: If any cycle found then the vertex matrix is inconsistent, otherwise consistent.

Step6: IMPR is consistent if both the vertex lower and the upper matrix is consistent; otherwise matrix 
is inconsistent.

Step7: If the IMPR matrix is inconsistent, then remove the minimum number of the path that covers the cycles.

An example illustrates the above-said method.

Example 1 Let us consider a decision making problem with four sets of alternatives $x_{i},(i=1,2,3,4)$. The decision maker judge these four alternatives by pairwise comparison and provides his/her judgement as follows: $\tilde{r}_{12}=(1 / 2,1 / 4), \tilde{r}_{13}=(2,1 / 8), \tilde{r}_{14}=(2 / 3,1 / 4), \tilde{r}_{23}=(5,1 / 7), \tilde{r}_{24}=(7 / 5,2 / 3), \tilde{r}_{34}=(6,1 / 7)$. The matrix representation is given by

$$
\tilde{R}_{1}=\left(\begin{array}{cccc}
(1,1) & \left(\frac{1}{2}, \frac{1}{4}\right) & \left(2, \frac{1}{8}\right) & \left(\frac{2}{3}, \frac{1}{4}\right) \\
\left(\frac{1}{4}, \frac{1}{2}\right) & (1,1) & \left(5, \frac{1}{7}\right) & \left(\frac{7}{5}, \frac{2}{3}\right) \\
\left(\frac{1}{8}, 2\right) & \left(\frac{1}{7}, 5\right) & (1,1) & \left(6, \frac{1}{7}\right) \\
\left(\frac{1}{4}, \frac{2}{3}\right) & \left(\frac{2}{3}, \frac{7}{5}\right) & \left(\frac{1}{7}, 6\right) & (1,1)
\end{array}\right)
$$

By using equation 8, the binary order pair vertex matrix is

$$
V=\left(\begin{array}{cccc}
(0,0) & (0,0) & (1,0) & (0,0) \\
(1,1) & (0,0) & (1,0) & (1,0) \\
(0,1) & (0,1) & (0,0) & (1,0) \\
(1,1) & (0,1) & (0,1) & (0,0)
\end{array}\right)
$$

The lower and upper vertex matrices are

$$
V_{L}=\left(\begin{array}{llll}
0 & 0 & 1 & 0 \\
1 & 0 & 1 & 1 \\
0 & 0 & 0 & 1 \\
1 & 0 & 0 & 0
\end{array}\right), V_{U}=\left(\begin{array}{llll}
0 & 0 & 0 & 0 \\
1 & 0 & 0 & 0 \\
1 & 1 & 0 & 0 \\
1 & 1 & 1 & 0
\end{array}\right)
$$

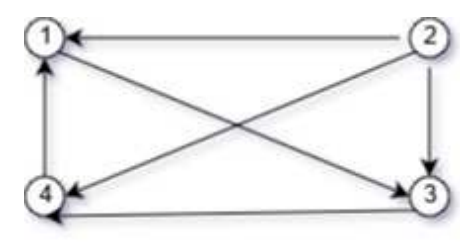

Fig. 1 Directed graph of $V_{L}$ of example 1

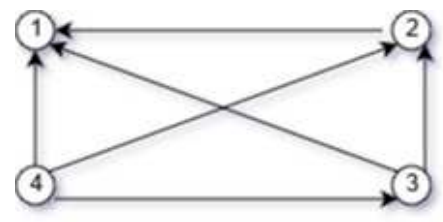

Fig. 2 Directed graph of $V_{U}$ of example 1

There is only one cycle of length 3 is present in the lower vertex matrix $V_{L}$. Directly we can found the cycle from figure 1 . To find cycles of length 3, we use step (2) to step (5) of odd-length algorithm (see algorithm 2), where $m=2$. The format of a cycle of length 3 is $\left(i_{0}-k-j_{0}\right)$.

To find the elements $i_{0}, k, j_{0}$, we need $V_{L}$ and $V_{L}^{2}$. For this $V_{L}^{2}$ as follows:

$$
V_{L}^{2}=\left(\begin{array}{llll}
0 & 0 & 0 & 1 \\
1 & 0 & 1 & 1 \\
1 & 0 & 0 & 0 \\
0 & 0 & 1 & 0
\end{array}\right)
$$

At the starting we have $i_{0}=1, k=3, j_{0}=4$, satisfying the step (2) of algorithm 2 of odd length cycle i.e $V_{L}(1,3)=1, V_{L}^{2}(3,1)=1, V_{L}(3,4)=1, V_{L}^{2}(4,3)=1$, and $V_{L}(4,1)=1$. Then the cycle of length 3 is $(1-3-4)$. There is no other cycle available in $V_{L}$. Similarly there is no cycle of any length in figure 2 . The lower vertex matrix $V_{L}$ is inconsistent and the upper vertex matrix $V_{U}$ is consistent. Therefore the 
Table 2

\begin{tabular}{|c|ccc|}
\hline Cycle & $(1,3)$ & $(1,4)$ & $(3,4)$ \\
\hline$(1-3-4)$ & 1 & 1 & 1 \\
\hline
\end{tabular}

complete IMPR is inconsistent. Apply an extinguishing cycles algorithm based on minimum covering sets to provide the cause of inconsistency (see Nishizawa, 1995). The cycle-arc incidence matrix in Table 2. From table 2, we get the several pairs of edges which cover the cycle. we have to chose the pair eliminate the cycle. In this example if we will change the pair of vertex from any one of them from $(3,4)$ to $(4,3)$ in the original IMPR $\tilde{R}_{1}$, then the both the lower and upper vertex matrix are consistent. Then IMPR $\tilde{R}_{1}$ is also consistent.

Example 2 Let us consider a decision-making problem with seven sets of alternatives $x_{i},(i=1,2, \cdots, 7)$. The DM judges these seven alternatives by pairwise comparison and provides his/her judgement. The matrix representation of the decision maker judgement is given by

$$
\tilde{R}_{2}=\left(\begin{array}{ccccccc}
(1,1) & \left(\frac{5}{3}, \frac{1}{4}\right) & \left(7, \frac{1}{9}\right) & \left(3, \frac{1}{7}\right) & \left(\frac{5}{3}, \frac{1}{7}\right) & \left(1, \frac{3}{5}\right) & \left(\frac{1}{4}, \frac{5}{3}\right) \\
\left(\frac{1}{4}, \frac{5}{3}\right) & (1,1) & \left(\frac{5}{3}, \frac{1}{4}\right) & \left(\frac{5}{3}, \frac{1}{4}\right) & \left(\frac{3}{5}, 1\right) & \left(\frac{1}{3}, 1\right) & \left(\frac{1}{4}, 3\right) \\
\left(\frac{1}{9}, 7\right) & \left(\frac{1}{4}, \frac{5}{3}\right) & (1,1) & \left(1, \frac{3}{5}\right) & \left(\frac{1}{4}, \frac{5}{3}\right) & \left(\frac{1}{3}, 3\right) & \left(\frac{1}{9}, 7\right) \\
\left(\frac{1}{7}, 3\right) & \left(\frac{1}{4}, \frac{5}{3}\right) & \left(\frac{3}{5}, 1\right) & (1,1) & \left(\frac{3}{5}, \frac{3}{5}\right) & \left(\frac{1}{3}, 3\right) & \left(\frac{1}{9}, 7\right) \\
\left(\frac{1}{7}, \frac{5}{3}\right) & \left(1, \frac{3}{5}\right) & \left(\frac{5}{3}, \frac{1}{4}\right) & \left(\frac{3}{5}, \frac{3}{5}\right) & (1,1) & \left(\frac{1}{3}, 3\right) & \left(\frac{1}{7}, 3\right) \\
\left(\frac{3}{5}, 1\right) & \left(1, \frac{1}{3}\right) & \left(3, \frac{1}{3}\right) & \left(3, \frac{1}{3}\right) & \left(3, \frac{1}{3}\right) & (1,1) & \left(\frac{1}{7}, 3\right) \\
\left(\frac{5}{3}, \frac{1}{4}\right) & \left(3, \frac{1}{4}\right) & \left(7, \frac{1}{9}\right) & \left(7, \frac{1}{9}\right) & \left(3, \frac{1}{7}\right) & \left(3, \frac{1}{7}\right) & (1,1)
\end{array}\right)
$$

Using 8 , the binary order pair vertex matrix is given by

$$
V=\left(\begin{array}{lllllll}
(0,0) & (1,0) & (1,0) & (1,0) & (1,0) & (0,0) & (0,1) \\
(0,1) & (0,0) & (1,0) & (1,0) & (0,0) & (0,0) & (0,1) \\
(0,1) & (0,1) & (0,0) & (0,0) & (0,1) & (0,1) & (0,1) \\
(0,1) & (0,1) & (1,1) & (0,0) & (0,0) & (0,1) & (0,1) \\
(0,1) & (1,0) & (1,0) & (1,1) & (0,0) & (0,1) & (0,1) \\
(0,1) & (1,0) & (1,0) & (1,0) & (1,0) & (0,0) & (0,1) \\
(1,0) & (1,0) & (1,0) & (1,0) & (1,0) & (1,0) & (0,0)
\end{array}\right)
$$

The above vertex matrix split into two binary array.

$$
V_{L}=\left(\begin{array}{lllllll}
0 & 1 & 1 & 1 & 1 & 0 & 0 \\
0 & 0 & 1 & 1 & 0 & 0 & 0 \\
0 & 0 & 0 & 0 & 0 & 0 & 0 \\
0 & 0 & 1 & 0 & 0 & 0 & 0 \\
0 & 1 & 1 & 1 & 0 & 0 & 0 \\
0 & 1 & 1 & 1 & 1 & 0 & 0 \\
1 & 1 & 1 & 1 & 1 & 1 & 0
\end{array}\right), \quad V_{U}=\left(\begin{array}{lllllll}
0 & 0 & 0 & 0 & 0 & 0 & 1 \\
1 & 0 & 0 & 0 & 0 & 0 & 1 \\
1 & 1 & 0 & 0 & 1 & 1 & 1 \\
1 & 1 & 1 & 0 & 0 & 1 & 1 \\
1 & 0 & 0 & 1 & 0 & 1 & 1 \\
1 & 0 & 0 & 0 & 0 & 0 & 1 \\
0 & 0 & 0 & 0 & 0 & 0 & 0
\end{array}\right)
$$

By applying algorithm 1 and 2, there is no cycle found in $V_{L}$, and one cycle of length three, i.e., $(3-5-4)$ is located in $V_{U}$. In order to find the cycle of length 3 in $V_{U}$ we use step (2) to step (5) of algorithm 2 , where $m=2$. The form of the cycle of length 3 is $\left(i_{0}-k-j_{0}\right)$. To locate these element we 


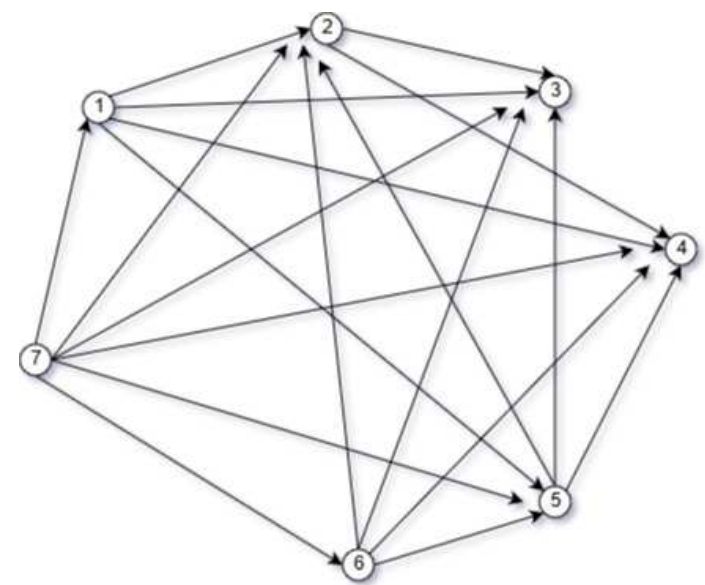

Fig. 3 Directed graph of $V_{L}$ of example 2

Table 3

\begin{tabular}{|c|ccc|}
\hline Cycle & $(3,4)$ & $(3,5)$ & $(4,5)$ \\
\hline$(3-5-4)$ & 1 & 1 & 1 \\
\hline
\end{tabular}

need $V_{U}$ and $V_{U}^{2}$. For this $V_{U}^{2}$ as follows:

$$
V_{U}^{2}=\left(\begin{array}{lllllll}
0 & 0 & 0 & 0 & 0 & 0 & 0 \\
0 & 0 & 0 & 0 & 0 & 0 & 1 \\
3 & 0 & 0 & 1 & 0 & 1 & 4 \\
3 & 1 & 0 & 0 & 1 & 1 & 4 \\
2 & 1 & 1 & 0 & 0 & 1 & 3 \\
0 & 0 & 0 & 0 & 0 & 0 & 1 \\
0 & 0 & 0 & 0 & 0 & 0 & 0
\end{array}\right)
$$

At the starting we have $i_{0}=3, k=5, j_{0}=4$, satisfying $V_{U}(3,5)=1, V_{U}^{2}(5,3)=1, V_{U}(5,4)=1$, $V_{U}^{2}(4,5)=1$, and $V_{U}(4,3)=1$. Then the cycle of length 3 is $(3-5-4)$. Similarly there is no cycle of any length in figure 4 . The lower vertex matrix $V_{L}$ is consistent and the upper vertex matrix $V_{U}$ is inconsistent. Therefore the complete IMPR is inconsistent.

We apply an extinguishing cycles algorithm based on minimum covering sets to provide the cause of inconsistency (see Nishizawa, 1995). The cycle-arc incidence matrix in Table 3. From table 3, we get the several pairs of edges which cover the cycle. we have to chose the pair eliminate the cycle. In this example if we will change the pair $(3,4)$ to $(4,3)$ in the original IMPR $\tilde{R}_{2}$, then both the lower and upper vertex matrix are consistent. Then IMPR $\tilde{R}_{2}$ is consistent.

The above said method is also applied in incomplete IMPRs scenario which is given in the following subsection.

\subsection{Consistency of incomplete IMPR}

In decision-making problem, the reality of the situation may prove that decision maker might not have a decent comprehension on a specific inquiry, and along these lines, he/she can not make an immediate correlation between two choices or criteria. Therefore, it is more fitting and adaptable to avoid a few similarities, and in those cases, the decision maker may like to express their judgments with incomplete preference relation. As indicated by the already talked about, the decision makers may not give exactly $\frac{n(n-1)}{2}$ judgments in practical decision making and the incomplete IMPR will be introduced. So, it is essential to investigate the incomplete preference relations as a useful tool in the decision-making 
problem, and many research results have been developed. Herrera-Viedma et al. (2007) proposed the meaning of incomplete preference relation. The idea of IMPRs is reached out to the circumstances where the preference data given by DM is incomplete.

Definition 6 (Jiang et al. 2015) An IMPR $\tilde{r}_{i j}=\left(\wp_{i j}, \Im_{i j}\right)_{n \times n}$ is called an incomplete IMPR if some elements in it are missing, and all available elements satisfy the characteristics of IMPR stated in Definition 3.

For an incomplete preference relation, it is very much essential for known elements to satisfy consistency. We are applying the same graphical approach in incomplete IMPRs scenario. For an incomplete comparison case, which includes unknown pairwise comparisons, we use the measure of inconsistency by the number of the cycle in the graph corresponding to known elements of the IMPR matrix. For the incomplete preference relation, if one wants to find the inconsistency, one condition is that the incomplete preference relation is acceptable, that is, in its directed graph, there is no isolated point.

Example 3 Let us consider a decision making problem with six sets of alternatives $x_{i},(i=1,2,3,4,5,6)$. The decision maker judge these six alternatives by pairwise comparison and provides his/her judgement as follows: $\tilde{r}_{12}=(1 / 5,2), \tilde{r}_{13}=(5,1 / 6), \tilde{r}_{15}=(1 / 9,8), \tilde{r}_{16}=(2,1 / 6), \tilde{r}_{24}=(1 / 4,1 / 5), \tilde{r}_{25}=(1 / 2,1 / 3)$, $\tilde{r}_{26}=(3,1 / 5), \tilde{r}_{35}=(6,1 / 7), \tilde{r}_{45}=(1 / 5,4)$. The matrix representation is given by

$$
\tilde{R}_{3}=\left(\begin{array}{cccccc}
(1,1) & \left(\frac{1}{5}, 2\right) & \left(5, \frac{1}{6}\right) & (*, *) & \left(\frac{1}{9}, 8\right) & \left(2, \frac{1}{6}\right) \\
\left(2, \frac{1}{5}\right) & (1,1) & (*, *) & \left(\frac{1}{4}, \frac{1}{5}\right) & \left(\frac{1}{2}, \frac{1}{3}\right) & \left(3, \frac{1}{5}\right) \\
\left(\frac{1}{6}, 5\right) & (*, *) & (1,1) & (*, *) & \left(6, \frac{1}{7}\right) & (*, *) \\
(*, *) & \left(\frac{1}{5}, \frac{1}{4}\right) & (*, *) & (1,1) & \left(\frac{1}{5}, 4\right) & (*, *) \\
\left(8, \frac{1}{9}\right) & \left(\frac{1}{3}, \frac{1}{2}\right) & \left(\frac{1}{7}, 6\right) & \left(4, \frac{1}{5}\right) & (1,1) & (*, *) \\
\left(\frac{1}{6}, 2\right) & \left(\frac{1}{5}, 3\right) & (*, *) & (*, *) & (*, *) & (1,1)
\end{array}\right)
$$

The order pair vertex matrix is obtained by using equation 8

$$
V=\left(\begin{array}{cccccc}
(0,0) & (0,1) & (1,0) & (0,0) & (0,1) & (1,0) \\
(1,0) & (0,0) & (0,0) & (0,0) & (0,0) & (1,0) \\
(0,1) & (0,0) & (0,0) & (0,0) & (1,0) & (0,0) \\
(0,0) & (1,1) & (0,0) & (0,0) & (0,1) & (0,0) \\
(1,0) & (1,1) & (0,1) & (1,0) & (0,0) & (0,0) \\
(0,1) & (0,1) & (0,0) & (0,0) & (0,0) & (0,0)
\end{array}\right)
$$

The lower and upper vertex matrices are given below

$$
V_{L}=\left(\begin{array}{llllll}
0 & 0 & 1 & 0 & 0 & 1 \\
1 & 0 & 0 & 0 & 0 & 1 \\
0 & 0 & 0 & 0 & 1 & 0 \\
0 & 1 & 0 & 0 & 0 & 0 \\
1 & 1 & 0 & 1 & 0 & 0 \\
0 & 0 & 0 & 0 & 0 & 0
\end{array}\right), V_{U}=\left(\begin{array}{llllll}
0 & 1 & 0 & 0 & 1 & 0 \\
0 & 0 & 0 & 0 & 0 & 0 \\
1 & 0 & 0 & 0 & 0 & 0 \\
0 & 1 & 0 & 0 & 1 & 0 \\
0 & 1 & 1 & 0 & 0 & 0 \\
1 & 1 & 0 & 0 & 0 & 0
\end{array}\right)
$$

To find the cycle of length 3 in the lower vertex matrix $V_{L}$, we need $V_{L}$ and $V_{L}^{2}$. For this $V_{L}^{2}$ as follows:

$$
V_{L}^{2}=\left(\begin{array}{llllll}
0 & 0 & 0 & 0 & 1 & 0 \\
0 & 0 & 1 & 0 & 0 & 1 \\
1 & 1 & 0 & 1 & 0 & 0 \\
1 & 0 & 0 & 0 & 0 & 1 \\
1 & 1 & 1 & 0 & 0 & 2 \\
0 & 0 & 0 & 0 & 0 & 0
\end{array}\right)
$$




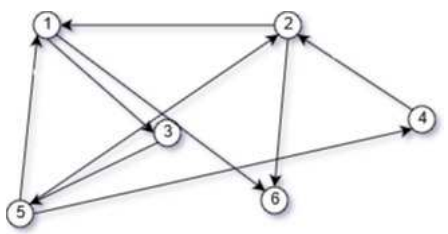

Fig. 5 Directed graph of $V_{L}$ of example 3

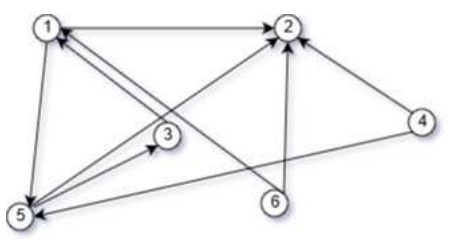

Fig. 6 Directed graph of $V_{U}$ of example 3

Table 4

\begin{tabular}{|c|ccc|}
\hline Cycle & $(1,5)$ & $(1,3)$ & $(3,5)$ \\
\hline$(1-3-5)$ & 1 & 1 & 1 \\
$(1-5-3)$ & 1 & 1 & 1 \\
\hline
\end{tabular}

At the starting we have $i_{0}=1, k=3, j_{0}=5$, satisfying the step (2) of algorithm 2 i.e $V_{L}(1,3)=1$, $V_{L}^{2}(3,1)=1, V_{L}(3,5)=1, V_{L}^{2}(5,3)=1$, and $V_{L}(5,1)=1$. Then the cycle of length 3 is $(1-3-5)$.

Similarly using algorithm 2, there is also one cycle of length 3 in $V_{U}$. Here also, for finding the cycle of length 3 in $V_{U}$, we need $V_{U}$ and $V_{U}^{2}$. For this $V_{U}^{2}$ as follows:

$$
V_{U}^{2}=\left(\begin{array}{llllll}
0 & 1 & 1 & 0 & 0 & 0 \\
0 & 0 & 0 & 0 & 0 & 0 \\
0 & 1 & 0 & 0 & 1 & 0 \\
0 & 1 & 1 & 0 & 0 & 0 \\
1 & 0 & 0 & 0 & 0 & 0 \\
0 & 1 & 0 & 0 & 1 & 0
\end{array}\right)
$$

Also we have $i_{0}=1, k=5, j_{0}=3$, satisfying $V_{U}(1,5)=1, V_{U}^{2}(5,1)=1, V_{U}(5,3)=1, V_{U}^{2}(3,5)=1$, and $V_{U}(3,1)=1$. Then the cycle of length 3 is $(1-5-3)$.

Both the lower and upper vertex matrix $V_{L}$, and $V_{U}$ is inconsistent. Therefore the incomplete IMPR is inconsistent. To suggest the cause of inconsistency, we apply an algorithm for extinguishing cycles based on minimum covering sets. The cycle-arc incidence matrix in Table 4.

From table 4, we get several pairs of the edges which cover the cycle. We have to chose the couple to eliminate the cycle. In this example, if we will change the pair $(3,5)$ to $(5,3)$ in the original IMPR $\tilde{R}_{4}$, then both the lower and upper vertex matrix are consistent. Then incomplete IMPR $\tilde{R}_{4}$ is also consistent. 
Example 4 Let us take an example of incomplete IMPRs of order $10 \times 10$ given as below

$$
\tilde{R}_{4}=\left[\begin{array}{ccccccccccc}
(1,1) & * & \left(\frac{1}{2}, \frac{1}{5}\right) & \left(\frac{1}{3}, 2\right) & \left(3, \frac{1}{6}\right) & * & * & \left(\frac{8}{3}, \frac{1}{8}\right) & \left(\frac{1}{7}, 4\right) & \left(5, \frac{1}{5}\right) \\
* & (1,1) & \left(\frac{2}{3}, \frac{6}{10}\right) & \left(\frac{1}{8}, \frac{1}{2}\right) & \left(\frac{1}{4}, 1\right) & * & \left(\frac{1}{5}, 5\right) & * & \left(\frac{1}{3}, 3\right) & * \\
\left(\frac{1}{5}, \frac{1}{2}\right) & \left(\frac{6}{10}, \frac{2}{3}\right) & (1,1) & \left(\frac{3}{5}, \frac{2}{3}\right) & * & \left(2, \frac{1}{2}\right) & \left(4, \frac{1}{8}\right) & \left(7, \frac{1}{8}\right) & \left(\frac{1}{8}, 5\right) & * \\
\left(2, \frac{1}{3}\right) & \left(\frac{1}{2}, \frac{1}{8}\right) & \left(\frac{2}{3}, \frac{3}{5}\right) & (1,1) & * & \left(3, \frac{1}{7}\right) & \left(5, \frac{1}{6}\right) & * & \left(2, \frac{1}{2}\right) & * \\
\left(\frac{1}{6}, 3\right) & \left(1, \frac{1}{4}\right) & * & * & (1,1) & \left(\frac{1}{8}, 7\right) & \left(8, \frac{1}{9}\right) & \left(\frac{1}{6}, 3\right) & * & \left(4, \frac{1}{5}\right) \\
* & * & \left(\frac{1}{2}, 2\right) & \left(\frac{1}{7}, 3\right) & \left(7, \frac{1}{8}\right) & (1,1) & * & * & \left(\frac{1}{5}, 4\right) & \left(2, \frac{1}{3}\right) \\
* & \left(5, \frac{1}{5}\right) & \left(\frac{1}{8}, 4\right) & \left(\frac{1}{6}, 5\right) & \left(\frac{1}{9}, 8\right) & * & (1,1) & \left(\frac{1}{9}, 6\right) & \left(\frac{1}{7}, 2\right) & \left(4, \frac{1}{8}\right) \\
\left(\frac{1}{8}, \frac{8}{3}\right) & * & \left(\frac{1}{8}, 7\right) & * & \left(3, \frac{1}{6}\right) & * & \left(6, \frac{1}{9}\right) & (1,1) & * & \left(6, \frac{1}{6}\right) \\
\left(4, \frac{1}{7}\right) & \left(3, \frac{1}{3}\right) & \left(5, \frac{1}{8}\right) & \left(\frac{1}{2}, 2\right) & * & \left(4, \frac{1}{5}\right) & \left(2, \frac{1}{7}\right) & * & (1,1) & \left(\frac{1}{3}, 2\right) \\
\left(\frac{1}{5}, 5\right) & * & * & * & \left(\frac{1}{5}, 4\right) & \left(\frac{1}{3}, 2\right) & \left(\frac{1}{8}, 4\right) & \left(\frac{1}{6}, 6\right) & \left(2, \frac{1}{3}\right) & (1,1)
\end{array}\right]
$$

The binary order pair vertex matrix is given by

$$
V=\left[\begin{array}{llllllllll}
(0,0) & (0,0) & (0,0) & (0,1) & (1,0) & (0,0) & (0,0) & (1,0) & (0,1) & (1,0) \\
(0,0) & (0,0) & (0,0) & (0,0) & (0,0) & (0,0) & (0,1) & (0,0) & (0,1) & (0,0) \\
(1,1) & (1,1) & 0,0) & (0,0) & (0,0) & (1,0) & (1,0) & (1,0) & (0,1) & (0,0) \\
(1,0) & (1,1) & (1,1) & (0,0) & (0,0) & (1,0) & (1,0) & (0,0) & (1,0) & (0,0) \\
(0,1) & (1,0) & (0,0) & (0,0) & (0,0) & (0,1) & (1,0) & (0,1) & (0,0) & (1,0) \\
(0,0) & (0,0) & (0,1) & (0,1) & (1,0) & (0,0) & (0,0) & (0,0) & (0,1) & (1,0) \\
(0,0) & (1,0) & (0,1) & (0,1) & (0,1) & (0,0) & (0,0) & (0,1) & (0,1) & (1,0) \\
(0,1) & (0,0) & (0,1) & (0,0) & (1,0) & (0,0) & (1,0) & (0,0) & (0,0) & (1,0) \\
(1,0) & (1,0) & (1,0) & (0,1) & (0,0) & (1,0) & (1,0) & (0,0) & (0,0) & (0,1) \\
(0,1) & (0,0) & (0,0) & (0,0) & (0,1) & (0,1) & (0,1) & (0,1) & (1,0) & (0,0)
\end{array}\right]
$$

The lower and upper vertex matrices are given below:

$$
V_{L}=\left[\begin{array}{llllllllll}
0 & 0 & 0 & 0 & 1 & 0 & 0 & 1 & 0 & 1 \\
0 & 0 & 0 & 0 & 0 & 0 & 0 & 0 & 0 & 0 \\
1 & 1 & 0 & 0 & 0 & 1 & 1 & 1 & 0 & 0 \\
1 & 1 & 1 & 0 & 0 & 1 & 1 & 0 & 1 & 0 \\
0 & 1 & 0 & 0 & 0 & 0 & 1 & 0 & 0 & 1 \\
0 & 0 & 0 & 0 & 1 & 0 & 0 & 0 & 0 & 1 \\
0 & 1 & 0 & 0 & 0 & 0 & 0 & 0 & 0 & 1 \\
0 & 0 & 0 & 0 & 1 & 0 & 1 & 0 & 0 & 1 \\
1 & 1 & 1 & 0 & 0 & 1 & 1 & 0 & 0 & 0 \\
0 & 0 & 0 & 0 & 0 & 0 & 0 & 0 & 1 & 0
\end{array}\right], V_{U}=\left[\begin{array}{llllllllll}
0 & 0 & 0 & 1 & 0 & 0 & 0 & 0 & 1 & 0 \\
0 & 0 & 0 & 0 & 0 & 0 & 1 & 0 & 1 & 0 \\
1 & 1 & 0 & 0 & 0 & 0 & 0 & 0 & 1 & 0 \\
0 & 1 & 1 & 0 & 0 & 0 & 0 & 0 & 0 & 0 \\
1 & 0 & 0 & 0 & 0 & 1 & 0 & 1 & 0 & 0 \\
0 & 0 & 1 & 1 & 0 & 0 & 0 & 0 & 1 & 0 \\
0 & 0 & 1 & 1 & 1 & 0 & 0 & 1 & 1 & 0 \\
1 & 0 & 1 & 0 & 0 & 0 & 0 & 0 & 0 & 0 \\
0 & 0 & 0 & 1 & 0 & 0 & 0 & 0 & 0 & 1 \\
1 & 0 & 0 & 0 & 1 & 1 & 1 & 1 & 0 & 0
\end{array}\right]
$$

There are three cycles of length 3 is found in the lower vertex matrix i.e. $(1-10-9),(6-10-9)$ and $(7-10-9)$. In order to find the cycle of length 3 in $V_{L}$ we use step (2) to step (5) of the algorithm 2, 
where $m=2$. The form of the cycle of length 3 is $\left(i_{0}-k-j_{0}\right)$. To find the element of the cycle, we need $V_{L}^{2}$ as follows:

$$
V_{L}^{2}=\left[\begin{array}{llllllllll}
0 & 1 & 0 & 0 & 1 & 0 & 2 & 0 & 1 & 2 \\
0 & 0 & 0 & 0 & 0 & 0 & 0 & 0 & 0 & 0 \\
0 & 1 & 0 & 0 & 3 & 0 & 1 & 1 & 0 & 4 \\
2 & 3 & 1 & 0 & 2 & 2 & 2 & 2 & 0 & 3 \\
0 & 1 & 0 & 0 & 0 & 0 & 0 & 0 & 1 & 1 \\
0 & 1 & 0 & 0 & 0 & 0 & 1 & 0 & 1 & 1 \\
0 & 0 & 0 & 0 & 0 & 0 & 0 & 0 & 1 & 0 \\
0 & 2 & 0 & 0 & 0 & 0 & 1 & 0 & 1 & 2 \\
1 & 2 & 0 & 0 & 2 & 1 & 1 & 2 & 0 & 3 \\
1 & 1 & 1 & 0 & 0 & 1 & 1 & 0 & 0 & 0
\end{array}\right]
$$

For the cycle $(1-10-9)$, we have $i_{0}=1, k=10, j_{0}=9$, satisfying $V_{L}(1,10)=1, V_{L}^{2}(10,1)>0$, $V_{L}(10,9)>0, V_{L}^{2}(9,10)>0$, and $V_{L}(9,1)=1$. Then the cycle of length 3 is $(1-10-9)$. Similarly for the cycle $(6-10-9)$, we have $i_{0}=6, k=10, j_{0}=9$, such that $V_{L}(6,10)>0, V_{L}^{2}(10,6)>0, V_{L}(10,9)>0$, $V_{L}^{2}(9,10)>0$, and $V_{L}(9,6)=1$. For the cycle $(7-10-9)$, we have $i_{0}=7, k=10, j_{0}=9$, such that $V(7,10)>0, V_{L}^{2}(10,7)>0, V_{L}(10,9)>0, V_{L}^{2}(9,10)>0$, and $V_{L}(9,7)=1$.

Next we try to find out the cycle of length 4 . The form of the cycle of length 4 is $\left(i_{0}-i_{1}-j_{0}-j_{1}\right)$. By using the algorithm 1, there are five cycle of length 4 that is $(5-10-9-6),(1-10-9-3),(3-$ $6-10-9),(3-7-10-9)$, and $(3-8-10-9)$. To find cycle of length 4 , we need $V_{L}^{2}$. For the cycle $(5-10-9-6)$, we have $i_{0}=5, j_{0}=9$, satisfying step (2) of algorithm 2 , since $V_{L}^{2}(5,9)>0$ and $V_{L}^{2}(9,5)>0$, and we have $i_{1}=10$ and $j_{1}=6$ satisfying step (3) of even length cycle algorithm 2 for $\alpha=1$. Since $V_{L}(5,10)=1, V_{L}^{2}(10,6)>0, V_{L}^{2}(6,10)>0$ and $V_{L}(9,6)=1$. After that it is confirm $V_{L}(10,9)=1$ and $V_{L}(6,5)=1$. Then we have cycle of length 4 is $(5-10-9-6)$. Similarly cycle of length 4 is $(1-10-9-3),(3-6-10-9),(3-7-10-9)$, and $(3-8-10-9)$. To find the cycle of 
length 5 , using the algorithm for odd cycles, we need $V_{L}, V_{L}^{2}$ and $V_{L}^{3}$. For this $V_{L}^{3}$ as follows:

$$
V_{L}^{3}=\left[\begin{array}{cccccccccc}
1 & 4 & 1 & 0 & 0 & 1 & 2 & 0 & 2 & 3 \\
0 & 0 & 0 & 0 & 0 & 0 & 0 & 0 & 0 & 0 \\
0 & 4 & 0 & 0 & 1 & 0 & 4 & 0 & 4 & 5 \\
1 & 5 & 0 & 0 & 6 & 1 & 5 & 3 & 3 & 10 \\
1 & 1 & 1 & 0 & 0 & 1 & 1 & 0 & 1 & 0 \\
1 & 2 & 1 & 0 & 0 & 1 & 1 & 0 & 1 & 1 \\
1 & 1 & 1 & 0 & 0 & 1 & 1 & 0 & 0 & 0 \\
1 & 2 & 1 & 0 & 0 & 1 & 1 & 0 & 2 & 1 \\
0 & 3 & 0 & 0 & 4 & 0 & 4 & 1 & 3 & 7 \\
1 & 2 & 0 & 0 & 2 & 1 & 1 & 2 & 0 & 3
\end{array}\right]
$$

The form of the cycle of length 5 is $\left(i_{0}-j_{1}-k-i_{1}-j_{0}\right)$. We have $i_{0}=3, k=7, j_{0}=9$, satisfying $V_{L}^{2}(3,7)>0, V_{L}^{3}(7,3)>0, V_{L}^{2}(7,9)>0, V_{L}^{3}(9,7)>0$ and $V_{L}(9,3)=1$. For $\alpha=1$, we have to find out $i_{1}$ and $j_{1}$ where $i_{1}=10$ and $j_{1}=8$ with $V_{L}^{3}(3,10)>0, V_{L}^{2}(10,3)>0, V_{L}(7,10)=1, V_{L}^{2}(9,8)>0$, $V_{L}^{3}(8,9)>0$ and $V_{L}(8,7)=1$. After that it is confirm $V_{L}(10,9)=1$ and $V_{L}(3,8)=1$. Then we have cycle of length 5 is $(3-8-7-10-9)$. Similarly cycle of length 5 are obtained i.e $(3-6-5-10-$ $9),(1-8-10-9-3),(1-5-10-9-3)$, and $(5-7-10-9-6)$.

Next we will try to find the cycle of length 6 . The form of the cycle of length 6 is $\left(i_{0}-i_{1}-i_{2}-j_{0}-j_{1}-j_{2}\right)$. We have $i_{0}=1 j_{0}=10$ with $V_{L}^{3}(1,10)>0, V_{L}^{3}(10,1)>0$. For $\alpha=1$ we have to find the vertices $i_{1}=5$ and $j_{1}=9$ satisfying step (3) of algorithm 1 satisfying $V_{L}(1,5)=1, V_{L}^{3}(5,9)>0, V_{L}^{3}(9,5)>0$, $V_{L}(10,9)=1$. Similarly, for $\alpha=2$ we have $i_{2}=7$ and $j_{2}=3$ satisfying step (4) of algorithm 2 i.e. $V_{L}(5,7)=1, V_{L}^{3}(7,3)>0, V_{L}^{3}(3,7)>0, V_{L}(9,3)=1$. After that it is confirm $V_{L}(7,10)=1$ and $V_{L}(3,1)=1$. Then we have cycle of length 6 is $(1-5-7-10-9-3)$. Other cycle of length 6 are given by $(1-8-7-10-9-3),(3-6-5-7-10-9),(3-8-5-7-10-9)$, and $(1-8-5-10-9-3)$. Similarly, one cycle of length 7 is found that is $(1-8-5-7-10-9-3)$. There is no cycle of length 8 , 9 and 10 is detected. For easy understanding, all the cycles of $V_{L}$ are presented in Table 5.

Table 5 All possible cycle of $V_{L}$

\begin{tabular}{|c|c|}
\hline Length & Cycles \\
\hline 3 & $(1109),(6109),(7109)$ \\
\hline 4 & $(51096),(11093),(36109),(37109),(38109)$ \\
\hline 5 & $(387109),(365109),(181093),(151093),(571096)$ \\
\hline 6 & $(1571093),(1871093),(3657109),(3857109),(1851093)$ \\
\hline 7 & $(18571093)$ \\
\hline $8 \sim 10$ & nothing \\
\hline
\end{tabular}

Similarly the possible cycles of the upper vertex matrix $V_{U}$ are given in table 6 .

Since both the lower and upper matrix $V_{L}$ and $V_{U}$ are inconsistent; therefore IMPR $\tilde{R}_{5}$ is also inconsistent.

To suggest the cause of inconsistency, we apply an algorithm for extinguishing cycles based on minimum covering sets (Nishizawa, 1995). In this example, from table 5 and 6 , the path $2-7,3-4,9-10$ which cover all the cycle. If we will change the pair $(3,4)$ to $(4,3),(2,7)$ to $(7,2),(9,10)$ to $(10,9)$, in the original IMPR $\tilde{R}_{5}$, then the lower vertex matrix $V_{L}$ and the upper vertex matrix $V_{U}$ are both consistent. Then IMPR $\tilde{R}_{5}$ is also consistent. 
Table 6 All possible cycle of $V_{U}$

\begin{tabular}{|c|c|}
\hline Length & Cycles \\
\hline 3 & $(143),(274),(273),(394),(1910),(6910)$, \\
\hline 4 & 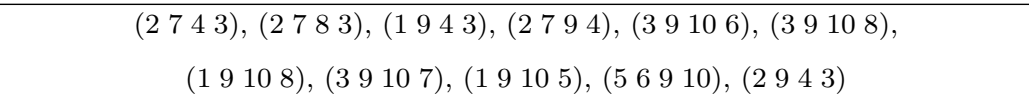 \\
\hline 5 & $\begin{array}{c}\text { (2 } 7563),(27564),(14275),(14273),(14278),(27583), \\
(27394),(142910),(391078),(191083),(191073), \\
(391074),(191063),(391064),(191058),(291083), \\
(291073),(291063),(291064),(291074),\end{array}$ \\
\hline 6 & $\begin{array}{c}(275643),(143275),(142758),(143278),(142783), \\
(194273),(2910743)(194278),(278394),(194275), \\
(275694),(2791083),(1429105),(2910564),(1439108), \\
(1910743),(1910643),(3910564)\end{array}$ \\
\hline 7 & $\begin{array}{c}(1427563),(1427583),(1942783),(2756394), \\
(1942758),(14291063),(2758394),(1943275),(2756943), \\
(14391058),(19105643),(14329108),(29105643),(14291058)\end{array}$ \\
\hline 8 & $\begin{array}{c}(19427563),(19427583),(19432758),(194327810) \\
(143291058),(143279108)\end{array}$ \\
\hline 9 & $(1427839105),(1910564278)$ \\
\hline 10 & $(19105643278)$ \\
\hline
\end{tabular}

\section{Isomorphism between Intuitionistic multiplicative preference relations(IMPR) and asymmetric multiplicative preference relation}

This section discusses the equivalence between the set of IMPR and the set of asymmetric multiplicative preference relation that leads to derive an asymmetric multiplicative preference relation from a given IMPR.

From the asymmetric multiplicative preference relation, we obtained the vertex matrix and checked for the consistency by using a directed graph in IMPR scenario.

Consider an IMPR

$$
\tilde{R}=\left(\begin{array}{cccccc}
\left(\wp_{11}, \Im_{11}\right) & \left(\wp_{12}, \Im_{12}\right) & \cdots & \left(\wp_{1 i}, \Im_{1 i}\right) & \cdots & \left(\wp_{1 n}, \Im_{1 n}\right) \\
\left(\wp_{21}, \Im_{21}\right) & \left(\wp_{22}, \Im_{22}\right) & \cdots & \left(\wp_{2 i}, \Im_{2 i}\right) & \cdots & \left(\wp_{2 n}, \Im_{2 n}\right) \\
\vdots & \vdots & \ddots & \vdots & \ddots & \vdots \\
\left(\wp_{i 1}, \Im_{i 1}\right) & \left(\wp_{i 2}, \Im_{i 2}\right) & \cdots & \left(\wp_{i i}, \Im_{i i}\right) & \cdots & \left(\wp_{i n}, \Im_{i n}\right) \\
\vdots & \vdots & \ddots & \vdots & \ddots & \vdots \\
\left(\wp_{n 1}, \Im_{n 1}\right) & \left(\wp_{n 2}, \Im_{n 2}\right) & \cdots & \left(\wp_{n i}, \Im_{n i}\right) & \cdots & \left(\wp_{n n}, \Im_{n n}\right)
\end{array}\right)
$$

The above relation can completely characterised using just its upper triangular part, because the intuitionistic multiplicative element $\left(\wp_{i j}, \Im_{i j}\right)$ is the mirror image of $\left(\wp_{j i}, \Im_{j i}\right)$.

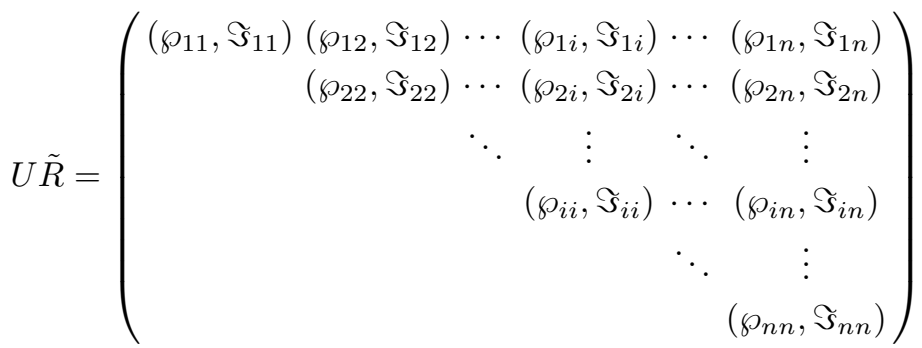


and this can be represented equivalently as the following MPR

$$
R=\left(\begin{array}{cccccc}
\wp_{11} & \wp_{12} & \cdots & \wp_{1 i} & \cdots & \wp_{1 n} \\
\Im_{12} & \wp_{22} & \cdots & \wp_{2 i} & \cdots & \wp_{2 n} \\
\vdots & \vdots & \ddots & \vdots & \ddots & \vdots \\
\Im_{1 i} & \Im_{2 i} & \cdots & \wp_{i i} & \cdots & \wp_{i n} \\
\vdots & \vdots & \ddots & \vdots & \ddots & \vdots \\
\Im_{1 n} & \Im_{2 n} & \cdots & \Im_{i n} & \cdots & \wp_{n n}
\end{array}\right)
$$

Since $\wp_{i j}=\Im_{j i}$ and $\Im_{i j}=\wp_{j i}$ then the above MPR becomes

$$
R=\left(\begin{array}{cccccc}
\wp_{11} & \wp_{12} & \cdots & \wp_{1 i} & \cdots & \wp_{1 n} \\
\wp_{21} & \wp_{22} & \cdots & \wp_{2 i} & \cdots & \wp_{2 n} \\
\vdots & \vdots & \ddots & \vdots & \ddots & \vdots \\
\wp_{i 1} & \wp_{i 2} & \cdots & \wp_{i i} & \cdots & \wp_{i n} \\
\vdots & \vdots & \ddots & \vdots & \ddots & \vdots \\
\wp_{n 1} & \wp_{n 2} & \cdots & \wp_{n i} & \cdots & \wp_{n n}
\end{array}\right)
$$

Let $\widetilde{Q}$ denote the set of IMPRs, where

$\widetilde{Q}=\left\{\tilde{R}=\left(\tilde{r}_{i j}\right) \mid \forall i, j: \tilde{r}_{i j}=\left(\wp_{i j}, \Im_{i j}\right), \wp_{i j}, \Im_{i j} \in[1 / 9,9], \wp_{i i}=\Im_{i i}=1, \wp_{i j}=\Im_{j i}, \wp_{j i}=\Im_{i j}, 0 \leq \wp_{i j} \Im_{i j} \leq 1\right\}$

and $\Re$ be the set of MPRs $\Re=\left\{R=\left(r_{i j}\right) \mid \forall i, j: r_{i j} \in[1 / 9,9]\right\}$. Define a mapping $f:[1 / 9,9] \times$ $[1 / 9,9] \rightarrow[1 / 9,9]$ by the function $f\left(x_{1}, x_{2}\right)=x_{1}$. We can define the following mapping, $F: \widetilde{Q} \rightarrow \Re$ between the set of IMPRs $\widetilde{Q}$ and the set of MPRs, $\Re$

$$
\left\{f\left(\tilde{r}_{i j}\right)\right\}=\left\{\wp_{i j}\right\} \text { i.e } R=F(\tilde{R})
$$

The following properties can be proved

Proposition 1 Function $F$ is well defined, i.e. For given $\tilde{R} \in \widetilde{Q} \Rightarrow f(\tilde{R}) \in \Re$.

Proof Let $\tilde{R}=\left(\tilde{r}_{i j}\right) \in \widetilde{Q}$.

Here $\tilde{r}_{i j}=\left(\wp_{i j}, \Im_{i j}\right) \Rightarrow f\left(\tilde{r}_{i j}\right)=f\left(\wp_{i j}, \Im_{i j}\right)=\wp_{i j} \in R$

Proposition 2 Function $F$ is one-one.

Proof Let $\tilde{R}_{1}=\left(\tilde{r}_{i j}^{1}\right)$ and $\tilde{R}_{2}=\left(\tilde{r}_{i j}^{2}\right)$ are IMPR such that $F\left(\tilde{R}_{1}\right)=F\left(\tilde{R}_{2}\right)$. Then we have that

$$
f\left(\tilde{r}_{i j}^{1}\right)=f\left(\tilde{r}_{i j}^{2}\right) \forall i, j \Leftrightarrow \wp_{i j}^{1}=\wp_{i j}^{2} \forall i, j .
$$

Because of the conditions of $\wp_{i j}^{1}=\Im_{j i}^{1}$ and $\wp_{i j}^{2}=\Im_{j i}^{2}$, then it is obvious that $\Im_{i j}^{1}=\Im_{i j}^{2}, \forall i, j$ Therefore, we have that

$$
\left(\wp_{i j}^{1}, \Im_{i j}^{1}\right)=\left(\wp_{i j}^{2}, \Im_{i j}^{2}\right) \Leftrightarrow \tilde{R}_{1}=\tilde{R}_{2} \forall i, j
$$

For the function to be onto, the following conditions to be verified:

$$
\forall R \in \Re \exists \tilde{R} \in \widetilde{Q}: F(\tilde{R})=R .
$$

By the definition of $F$ and $\widetilde{Q}$, we have that $R=\left(r_{i j}\right)=\left(\wp_{i j}\right)$ satisfies:

$$
0 \leq r_{i j} r_{j i}=\wp_{i j} \wp_{j i} \leq 1 .
$$

Thus $R$ is asymmetric multiplicative preference relation that proves the range of the function $F$ is the subset of MPRs which are asymmetric. 
Theorem 1 The set of intuitionistic multiplicative preference relations is isomorphic to set of asymmetric multiplicative preference relations.

Proof We know that when $\tilde{R} \in \widetilde{Q}$ has hesitancy degree always zero, then we have that:

$$
\wp_{i j} \Im_{i j}=1, \forall i, j
$$

In this case, $F(\tilde{R})=R$ is also reciprocal, i.e. $r_{i j} r_{j i}=1 \forall i, j$. The proof of this is quite simple as we have the following:

$$
\forall i, j: r_{i j}=f\left(\tilde{r}_{i j}\right)=\wp_{i j} \wedge r_{j i}=f\left(\tilde{r}_{j i}\right)=\wp_{j i}
$$

Since $\tilde{R} \in \widetilde{Q}$ then we have that $\wp_{j i}=\Im_{i j} \forall i, j$ and by using equation 9 it is $r_{i j} r_{j i}=\wp_{i j} \wp_{j i}=\wp_{i j} \Im_{i j}=$ $1 \forall i, j$.

Example 5 Isomorphic asymmetric multiplicative preference relation of IMPR of example 2 is

$$
I_{\tilde{R}_{2}}=\left(\begin{array}{ccccccc}
1 & \frac{5}{3} & 7 & 3 & \frac{5}{3} & 1 & \frac{1}{4} \\
\frac{1}{4} & 1 & \frac{5}{3} & \frac{5}{3} & \frac{3}{5} & \frac{1}{3} & \frac{1}{4} \\
\frac{1}{9} & \frac{1}{4} & 1 & 1 & \frac{1}{4} & \frac{1}{3} & \frac{1}{9} \\
\frac{1}{7} & \frac{1}{4} & \frac{3}{5} & 1 & \frac{3}{5} & \frac{1}{3} & \frac{1}{9} \\
\frac{1}{7} & 1 & \frac{5}{3} & \frac{3}{5} & 1 & \frac{1}{3} & \frac{1}{7} \\
\frac{3}{5} & 1 & 3 & 3 & 3 & 1 & \frac{1}{7} \\
\frac{5}{3} & 3 & 7 & 7 & 3 & 3 & 1
\end{array}\right)
$$

The vertex matrix of above asymmetric MPR is

$$
V=\left(\begin{array}{lllllll}
0 & 1 & 1 & 1 & 1 & 0 & 0 \\
0 & 0 & 1 & 1 & 0 & 0 & 0 \\
0 & 0 & 0 & 0 & 0 & 0 & 0 \\
0 & 0 & 0 & 0 & 0 & 0 & 0 \\
0 & 0 & 1 & 0 & 0 & 0 & 0 \\
0 & 0 & 1 & 1 & 1 & 0 & 0 \\
1 & 1 & 1 & 1 & 1 & 1 & 0
\end{array}\right)
$$

By using the algorithm 1 and 2 of odd and even length cycle, there is no cycle is present in the vertex matrix $V$. Hence $\tilde{R}_{2}$ is consistent. Also we can directly see from the figure 9 .

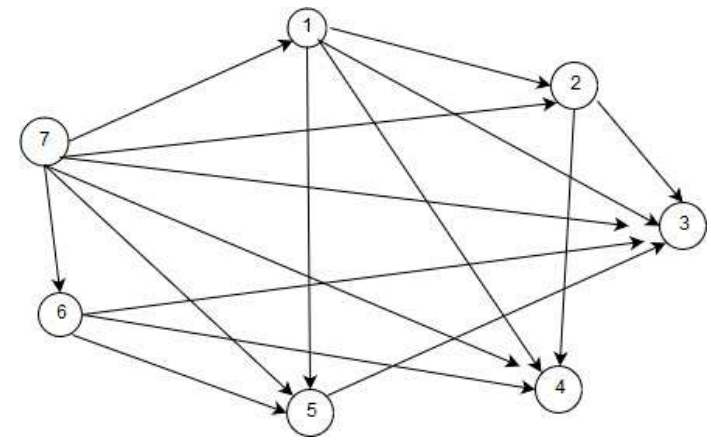

Fig. 9 Directed graph of example 5

Also same isomorphism approach is applied in incomplete IMPRs scenario. 
Example 6 Isomorphic asymmetric MPR of of example 3 is

$$
I_{\tilde{R}_{3}}=\left(\begin{array}{cccccc}
1 & \frac{1}{5} & 5 & * & \frac{1}{9} & 2 \\
2 & 1 & * & \frac{1}{4} & \frac{1}{2} & 3 \\
\frac{1}{6} & * & 1 & * & 6 & * \\
* & \frac{1}{5} & * & 1 & \frac{1}{5} & * \\
8 & \frac{1}{3} & \frac{1}{7} & 4 & 1 & * \\
\frac{1}{6} & \frac{1}{5} & * & * & * & 1
\end{array}\right)
$$

Vertex matrix of the asymmetric $\operatorname{MPR}, I_{\tilde{R}_{3}}$ is

$$
V=\left(\begin{array}{llllll}
0 & 0 & 1 & 0 & 0 & 1 \\
1 & 0 & 0 & 0 & 0 & 1 \\
0 & 0 & 0 & 0 & 1 & 0 \\
0 & 0 & 0 & 0 & 0 & 0 \\
1 & 0 & 0 & 1 & 0 & 0 \\
0 & 0 & 0 & 0 & 0 & 0
\end{array}\right)
$$

Using the algorithm 1 and 2 , the is one cycle of length 3 i.e. $(1-3-5)$ is present. Therefore, $\tilde{R}_{3}$ is inconsistent. In this example, if we will change the pair $(3,5)$ to $(5,3)$ in the original incomplete IMPR

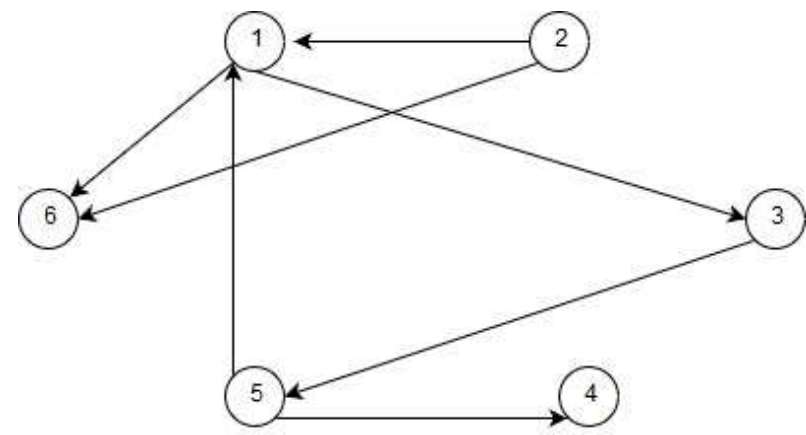

Fig. 10 Directed graph of 6

$\tilde{R}_{3}$, then it is consistent.

Example 7 Isomorphic asymmetric MPR of example 4 is

$$
I_{\tilde{R}_{4}}=\left(\begin{array}{cccccccccc}
1 & * & \frac{1}{2} & \frac{1}{3} & 3 & * & * & \frac{8}{3} & \frac{1}{7} & 5 \\
* & 1 & \frac{2}{3} & \frac{1}{8} & \frac{1}{4} & * & \frac{1}{5} & * & \frac{1}{3} & * \\
\frac{1}{5} & \frac{6}{10} & 1 & \frac{3}{5} & * & 2 & 4 & 7 & \frac{1}{8} & * \\
2 & \frac{1}{2} & \frac{2}{3} & 1 & * & 3 & 5 & * & 2 & * \\
\frac{1}{6} & 1 & * & * & 1 & \frac{1}{8} & 8 & \frac{1}{6} & * & 4 \\
* & * & \frac{1}{2} & \frac{1}{7} & 7 & 1 & * & * & \frac{1}{5} & 2 \\
* & 5 & \frac{1}{8} & \frac{1}{6} & \frac{1}{9} & * & 1 & \frac{1}{9} & \frac{1}{7} & 4 \\
\frac{1}{8} & * & \frac{1}{8} & * & 3 & * & 6 & 1 & * & 6 \\
4 & 3 & 5 & \frac{1}{2} & * & 4 & 2 & * & 1 & \frac{1}{3} \\
\frac{1}{5} & * & * & * & \frac{1}{5} & \frac{1}{3} & \frac{1}{8} & \frac{1}{6} & 2 & 1
\end{array}\right)
$$


vertex matrix of $I_{\tilde{R}_{4}}$ is

$$
V=\left(\begin{array}{llllllllll}
0 & 0 & 0 & 0 & 1 & 0 & 0 & 1 & 0 & 1 \\
0 & 0 & 0 & 0 & 0 & 0 & 0 & 0 & 0 & 0 \\
0 & 0 & 0 & 0 & 0 & 1 & 1 & 1 & 0 & 0 \\
1 & 0 & 0 & 0 & 0 & 1 & 1 & 0 & 1 & 0 \\
0 & 0 & 0 & 0 & 0 & 0 & 1 & 0 & 0 & 1 \\
0 & 0 & 0 & 0 & 1 & 0 & 0 & 0 & 0 & 1 \\
0 & 1 & 0 & 0 & 0 & 0 & 0 & 0 & 0 & 1 \\
0 & 0 & 0 & 0 & 1 & 0 & 1 & 0 & 0 & 1 \\
1 & 1 & 1 & 0 & 0 & 1 & 1 & 0 & 0 & 0 \\
0 & 0 & 0 & 0 & 0 & 0 & 0 & 0 & 1 & 0
\end{array}\right)
$$

By using the algorithm of odd and even length cycle 1 and 2, the corresponding cycles are given in table 6.

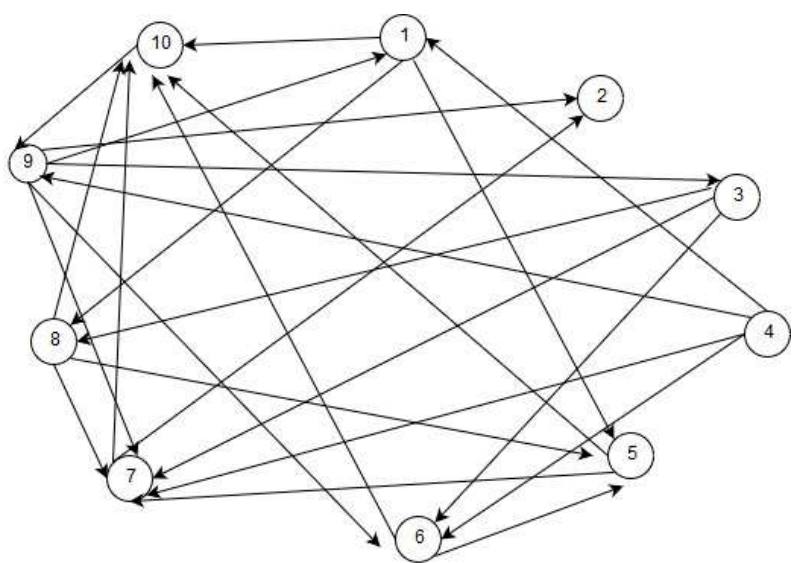

Fig. 11 Directed graph of Example 7

Table 7 All possible cycle of $V$

\begin{tabular}{|c|c|}
\hline Length & Cycles \\
\hline 3 & $(1109),(6109),(7109)$ \\
\hline 4 & $(51096),(18109),(15109),(37109),(38109),(36109)$ \\
\hline 5 & $(387109),(365109),(187109),(185109),(571096),(385109),(157109)$ \\
\hline 6 & $(1857109),(5710936),(3657109),(3857109),(5710938)$ \\
\hline $7 \sim 10$ & nothing \\
\hline
\end{tabular}

Therefore, $\tilde{R}_{5}$ is inconsistent. In table 7 it is conclude the path $9-10$ that cover all the cycle. If we will change the pair $(9,10)$ to $(10,9)$, in the original IMPR $\tilde{R}_{5}$, then $\tilde{R}_{5}$ is consistent.

\subsection{Comparison between two different Approaches:}

In example $2, \tilde{R}_{2}$ is inconsistent according to approach 1 has no cycle found in the lower vertex matrix and one cycle of length $3(3-5-4)$ found in the upper vertex matrix. But it is consistent according to approach 2. To suggest the cause of inconsistency in approach 1 , we will change the path $(3,4)$ to $(4,3)$ 
in the original IMPR $\tilde{R}_{2}$; then both the lower and upper vertex is consistent. Therefore $\tilde{R}_{2}$ is consistent. From the two approaches, we have concluded that the second approach is better than approach 1 .

In example $3, \tilde{R}_{3}$ is inconsistent according to approach 1 has one cycle found i.e. $(1-3-5)$ in the lower vertex matrix and one cycle of length 3 , i.e. $(1-5-3)$ is found in the upper vertex matrix. Also according to approach $2 \tilde{R}_{3}$ is inconsistent where one cycle is found in isometric matrix i.e. $(1-3-5)$. In both the approach the path $3-5$ which cover the cycle. We have to choose the couple to eliminate the cycle. In this case, if we will change the pair $(3,5)$ to $(5,3)$ in the original IMPR $\tilde{R}_{4}$, then both the lower and upper vertex is consistent. Then IMPR $\tilde{R}_{4}$ is also consistent. Therefore approach 2 is better than approach 1 because less no of the cycle is found in approach 2 .

In example 4, According to approach 1, 19 cycles are found in the lower vertex matrix $V_{L}$ and 78 cycles are found in $V_{U}$ that are given in table 5 and 6 . Similarly, according to approach 2, 21 cycles are found that are given in table 7 . In approach 1 , we conclude that if we change the path $9-10,4-3$, and $2-7$, then the IMPR $\tilde{R}_{4}$ is consistent. But according to approach 2 , if we will change the pair $(9,10)$ to $(10,9)$, in the original IMPR $\tilde{R}_{5}$, then $\tilde{R}_{5}$ is consistent. Therefore, approach 2 is better than approach 1 .

\section{Comparative analysis with the existing methods}

Lot of research has been developed in literature in the area of MPR, incomplete MPR, IMPR, incomplete IMPRs, and their consistency. In this section we have compare our methodology with these exsting techniques in literature.

\section{Comparison with Xu et al. (2013)}

Chiclana et al. (2001) studied the transformation function between reciprocal MPR with values in the interval scale $(1 / 9,9)$, and reciprocal FPRs with values in $[0,1]$.

Proposition 3 (Chiclana et al. (2001)) Suppose that $X=\left\{x_{1}, x_{2}, \cdots, x_{n}\right\}$, and associated to it a reciprocal multiplicative preference relation $A=\left(a_{i j}\right)_{n \times n}$ with $a_{i j} \in[1 / 9,9]$, then the corresponding reciprocal fuzzy preference relation, $P=\left(p_{i j}\right)_{n \times n}$ with $p_{i j} \in[0,1]$, associated with $A$ is given as follows $p_{i j}=\frac{1}{2}\left(1+\log _{9} a_{i j}\right)$

After spliting the IMPR into two MPRs, the two MPRs is converted into two FPRs using the proposition 3. Xu et al. (2013) proposed the ordinal consistency index to measure the degree of ordinal consistency of a FPR, which is to count the unreasonable 3-cycles in a directed graph that represents the FPR. Using this concept we check the consistency of IMPR. Let us take example 1

$$
\tilde{R}_{1}=\left(\begin{array}{cccc}
(1,1) & \left(\frac{1}{2}, \frac{1}{4}\right) & \left(2, \frac{1}{8}\right) & \left(\frac{2}{3}, \frac{1}{4}\right) \\
\left(\frac{1}{4}, \frac{1}{2}\right) & (1,1) & \left(5, \frac{1}{7}\right) & \left(\frac{7}{5}, \frac{2}{3}\right) \\
\left(\frac{1}{8}, 2\right) & \left(\frac{1}{7}, 5\right) & (1,1) & \left(6, \frac{1}{7}\right) \\
\left(\frac{1}{4}, \frac{2}{3}\right) & \left(\frac{2}{3}, \frac{7}{5}\right) & \left(\frac{1}{7}, 6\right) & (1,1)
\end{array}\right)
$$

This IMPR $\tilde{R}_{1}$ is split into two MPRs using equation 6 that is given below

$$
A_{1}=\left[\begin{array}{llll}
1 & \frac{1}{2} & 2 & \frac{2}{3} \\
2 & 1 & 5 & \frac{7}{5} \\
\frac{1}{2} & \frac{1}{5} & 1 & 6 \\
\frac{3}{2} & \frac{5}{7} & \frac{1}{6} & 1
\end{array}\right] \quad A_{2}=\left[\begin{array}{llll}
1 & \frac{1}{4} & \frac{1}{8} & \frac{1}{4} \\
4 & 1 & \frac{1}{7} & \frac{2}{3} \\
8 & 7 & 1 & \frac{1}{7} \\
4 & \frac{3}{2} & 7 & 1
\end{array}\right]
$$

Using the proposition 3 the above two MPRs converted into two FPRs i.e $P_{1}$ and $P_{2}$ given by 


$$
P_{1}=\left[\begin{array}{cccc}
0.5 & 0.3423 & 0.6577 & 0.4077 \\
0.6577 & 0.5 & 0.8662 & 0.5766 \\
0.3423 & 0.1338 & 0.5 & 0.9077 \\
0.5923 & 0.4234 & 0.0923 & 0.5
\end{array}\right] \quad P_{2}=\left[\begin{array}{cccc}
0.5 & 0.1845 & 0.0268 & 0.1845 \\
0.8155 & 0.5 & 0.0572 & 0.4077 \\
0.9732 & 0.9428 & 0.5 & 0.0572 \\
0.8155 & 0.5923 & 0.9428 & 0.5
\end{array}\right]
$$

According to $\mathrm{Xu}$ et al. (2013) the adjacency matrix of $P_{1}$ is

$$
E=\left[\begin{array}{llll}
0 & 0 & 1 & 0 \\
1 & 0 & 1 & 1 \\
0 & 0 & 0 & 1 \\
1 & 0 & 0 & 0
\end{array}\right] \quad E^{3}=\left[\begin{array}{llll}
1 & 0 & 0 & 0 \\
1 & 0 & 1 & 1 \\
0 & 0 & 1 & 0 \\
0 & 0 & 0 & 1
\end{array}\right]
$$

The Hadamard product of $E^{2}$ and $E^{T}$ is denoted by $B$ that is given below

$$
B=\left[\begin{array}{llll}
1 & 0 & 0 & 0 \\
1 & 0 & 1 & 1 \\
0 & 0 & 1 & 0 \\
0 & 0 & 0 & 1
\end{array}\right]
$$

\begin{tabular}{|c|c|c|c|c|c|c|c|c|c|c|c|c|c|}
\hline 1 & 2 & 3 & & 1 & 2 & 4 & & 2 & 3 & 4 & & 1 & 3 \\
\hline $1 / 0$ & 0 & $0)$ & 1 & 0 & 0 & $1)$ & 2 & 0 & 0 & 0 & 1 & 0 & 0 \\
\hline 0 & 0 & 0 & 2 & 0 & 0 & 0 & 3 & 0 & 0 & 0 & 3 & 1 & 0 \\
\hline$\backslash_{1}$ & 0 & 0 & 4 & 0 & 0 & $0)$ & 4 & $\backslash 0$ & 1 & $0)$ & 4 & $\backslash 0$ & 1 \\
\hline
\end{tabular}

Thus $\frac{\operatorname{trac}\left(E^{3}\right)}{3}=\frac{\sum_{i=1}^{4} \sum_{j=1}^{4} b_{i j}}{3}=1$. Then there is one 3 -cycle in the FPR $P_{1}$. To identify the cycle we will collect all the $3 \times 3$ pricipal sub-matrices of $\mathrm{B}$ are

There does not exist a zero row or column in the principal sub matrix $B[1,3,4]$. Therefore, there is one 3cycle in diagraph of $P_{1}$ i.e. $V_{1} \rightarrow V_{4} \rightarrow V_{3} \rightarrow V_{1}$ (see figure 12). The inconsistent entries are $r_{31}^{(0)}, r_{14}^{(0)}, r_{43}^{(0)}$. In our method also we got same inconsistent entries. Thus $P_{1}$ is order inconsistent and each entries appear once time in the cycle. So, $r_{31}^{(0)}=0.3423, r_{14}^{(0)}=0.4077, r_{43}^{(0)}=0.0923$. According to Xu et al.(2013), we choose the entry that is closer to 0.5 . From these above three value $r_{14}^{(0)}=0.4077$ is near to 0.5 . Therefore, take $r_{14}^{(1)}=1-0.4077=0.5923$, and $r_{41}^{(1)}=0.4077$ in $P_{1}$ matrix then apply same procedure it becomes consistent. Similarly, the adjacency matrix corresponding to $P_{2}$ is given by

$$
E=\left[\begin{array}{llll}
0 & 0 & 0 & 0 \\
1 & 0 & 0 & 0 \\
1 & 1 & 0 & 0 \\
1 & 1 & 1 & 0
\end{array}\right]
$$

The Hadamard product of $E^{2}$ and $E^{T}$ is denoted by $B$ that is given below

$$
B=\left[\begin{array}{llll}
0 & 0 & 0 & 0 \\
0 & 0 & 0 & 0 \\
0 & 0 & 0 & 0 \\
0 & 0 & 0 & 0
\end{array}\right]
$$

Here, $\frac{\sum_{i=1}^{4} \sum_{j=1}^{4} b_{i j}}{3}=0$ then there is no 3 -cycles are found in $P_{2}$ matrix. In our method also, the MPR $A_{2}$ has no cycles. Therefore $P_{2}$ is consistent. Since, both the FPRs $P_{1}, P_{2}$ are consistent, then the IMPR is consistent.

Let us take another example 2 


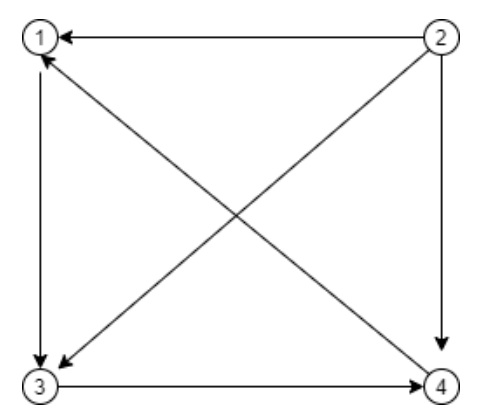

Fig. 12 Diagraph of FPR $P_{1}$ of example 1

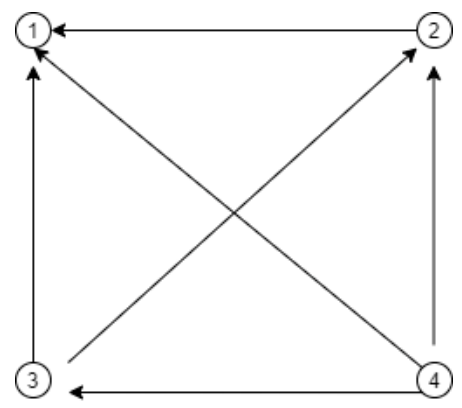

Fig. 13 Diagraph of FPR $P_{2}$ of example 1

$$
\tilde{R}_{2}=\left(\begin{array}{ccccccc}
(1,1) & \left(\frac{5}{3}, \frac{1}{4}\right) & \left(7, \frac{1}{9}\right) & \left(3, \frac{1}{7}\right) & \left(\frac{5}{3}, \frac{1}{7}\right) & \left(1, \frac{3}{5}\right) & \left(\frac{1}{4}, \frac{5}{3}\right) \\
\left(\frac{1}{4}, \frac{5}{3}\right) & (1,1) & \left(\frac{5}{3}, \frac{1}{4}\right) & \left(\frac{5}{3}, \frac{1}{4}\right) & \left(\frac{3}{5}, 1\right) & \left(\frac{1}{3}, 1\right) & \left(\frac{1}{4}, 3\right) \\
\left(\frac{1}{9}, 7\right) & \left(\frac{1}{4}, \frac{5}{3}\right) & (1,1) & \left(1, \frac{3}{5}\right) & \left(\frac{1}{4}, \frac{5}{3}\right) & \left(\frac{1}{3}, 3\right) & \left(\frac{1}{9}, 7\right) \\
\left(\frac{1}{7}, 3\right) & \left(\frac{1}{4}, \frac{5}{3}\right) & \left(\frac{3}{5}, 1\right) & (1,1) & \left(\frac{3}{5}, \frac{3}{5}\right) & \left(\frac{1}{3}, 3\right) & \left(\frac{1}{9}, 7\right) \\
\left(\frac{1}{7}, \frac{5}{3}\right) & \left(1, \frac{3}{5}\right) & \left(\frac{5}{3}, \frac{1}{4}\right) & \left(\frac{3}{5}, \frac{3}{5}\right) & (1,1) & \left(\frac{1}{3}, 3\right) & \left(\frac{1}{7}, 3\right) \\
\left(\frac{3}{5}, 1\right) & \left(1, \frac{1}{3}\right) & \left(3, \frac{1}{3}\right) & \left(3, \frac{1}{3}\right) & \left(3, \frac{1}{3}\right) & (1,1) & \left(\frac{1}{7}, 3\right) \\
\left(\frac{5}{3}, \frac{1}{4}\right) & \left(3, \frac{1}{4}\right) & \left(7, \frac{1}{9}\right) & \left(7, \frac{1}{9}\right) & \left(3, \frac{1}{7}\right) & \left(3, \frac{1}{7}\right) & (1,1)
\end{array}\right)
$$

This IMPR $R_{2}$ is split into two MPRs using equation 6 that is given below

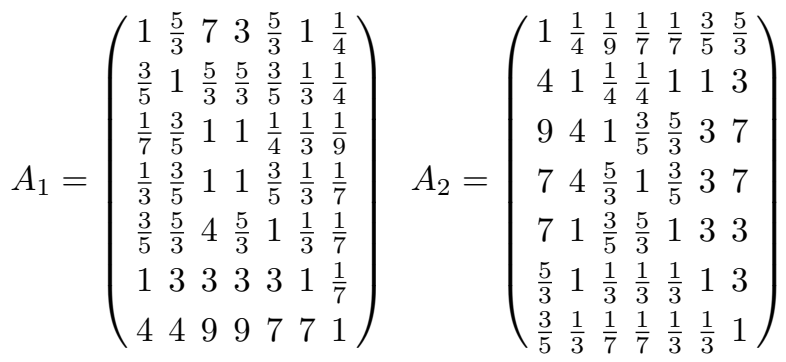

The MPRs converted into FPRs that is $P_{1}$ and $P_{2}$ is given by

$$
P_{1}=\left[\begin{array}{ccccccc}
0.5 & 0.6162 & 0.9428 & 0.75 & 0.6162 & 0.5 & 0.1845 \\
0.3838 & 0.5 & 0.6162 & 0.6162 & 0.3838 & 0.25 & 0.1845 \\
0.0572 & 0.3838 & 0.5 & 0.5 & 0.1845 & 0.25 & 0 \\
0.25 & 0.3838 & 0.5 & 0.5 & 0.3838 & 0.25 & 0 \\
0.3838 & 0.6162 & 0.8155 & 0.6162 & 0 & 0.25 & 0.0572 \\
0.5 & 0.75 & 0.75 & 0.75 & 0.75 & 0.5 & 0.0572 \\
0.8155 & 0.8155 & 1 & 1 & 0.9428 & 0.9428 & 0.5
\end{array}\right]
$$$$
P_{2}=\left[\begin{array}{cccccccc}
0.5 & 0.1845 & 0 & 0.0572 & 0.0572 & 0.3838 & 0.6162 \\
0.8155 & 0.5 & 0.1845 & 0.1845 & 0.5 & 0.5 & 0.75 \\
1 & 0.8155 & 0.5 & 0.3838 & 0.6162 & 0.75 & 0.9428 \\
0.9428 & 0.8155 & 0.6162 & 0.5 & 0.3838 & 0.75 & 0.9428 \\
0.9428 & 0.5 & 0.3838 & 0.6162 & 0.5 & 0.75 & 0.75 \\
0.6162 & 0.5 & 0.25 & 0.25 & 0.25 & 0.5 & 0.75 \\
0.3838 & 0.25 & 0.0572 & 0.0572 & 0.25 & 0.25 & 0.5
\end{array}\right]
$$

The adjacency matrix of the FPR $P_{1}$ is

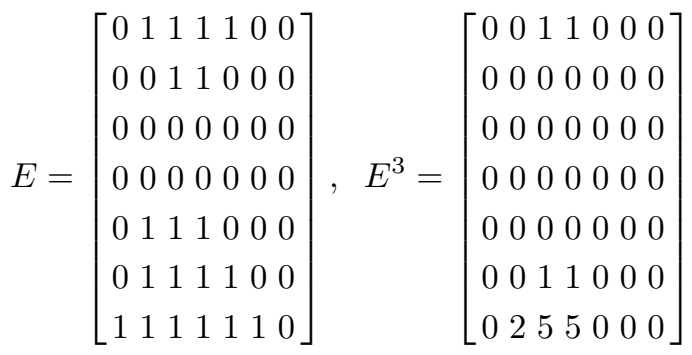


Thus $\frac{\operatorname{trac}\left(E^{3}\right)}{3}=0$. There is no 3 -cycles. Similarly, the adjacency matrix of $P_{2}$ matrix is

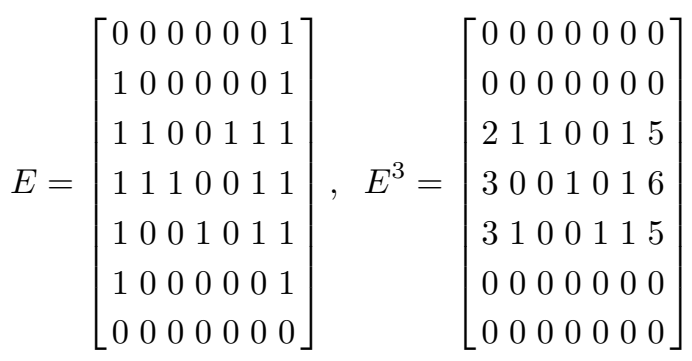

The Hadamard product of $E^{2}$ and $E^{T}$ is denoted by $B$ that is given below

$$
B=\left[\begin{array}{lllllll}
0 & 0 & 0 & 0 & 0 & 0 & 0 \\
0 & 0 & 0 & 0 & 0 & 0 & 0 \\
0 & 0 & 0 & 1 & 0 & 0 & 0 \\
0 & 0 & 0 & 0 & 1 & 0 & 0 \\
0 & 0 & 1 & 0 & 0 & 0 & 0 \\
0 & 0 & 0 & 0 & 0 & 0 & 0 \\
0 & 0 & 0 & 0 & 0 & 0 & 0
\end{array}\right]
$$

Thus $\frac{\operatorname{trac}\left(E^{3}\right)}{3}=\frac{\sum_{i=1}^{4} \sum_{j=1}^{4} b_{i j}}{3}=1$. Then there is one 3 -cycle. To identify the cycle we have $35,3 \times 3$ principal sub-matrices of $\mathrm{B}$. Out of these, the principal sub matrix $B[3,4,5]$ has no zero row and column. Therefore, there are one 3-cycles in diagraph of adjacency matrix of $P_{2}$, i.e. $V_{1} \rightarrow V_{4} \rightarrow V_{3} \rightarrow V_{1}$. The inconsistent entries are $r_{34}^{(0)}, r_{45}^{(0)}, r_{53}^{(0)}$. In our method also we got same inconsistent entries. Thus $P_{1}$ is order inconsistent and each entries appear once time in the cycle. So, $r_{34}^{(0)}=0.3838, r_{45}^{(0)}=0.3838, r_{53}^{(0)}=$ 0.0.3838. According to Xu et al. (2013), we choose the entry that is closer to 0.5. From these above three value take any one of them. Take $r_{34}^{(1)}=1-0.3838=0.6162$ and $r_{43}^{(1)}=0.3838$ in $P_{1}$ matrix then apply same procedure it becomes consistent.

Both the methods are equivalent. we are getting the same cycles in both the methods. In our method, we can take any order of matrix and check the consistency. But Xu et al. (2013) methods is complicated to find the inconsistency entries from the principal sub-matrix as for $n$-order matrix the number of principal sub-matrices of order 3 are $n_{C_{3}}$.

\section{Comparison with Jiang et al. (2015)}

Jiang et al. (2015) proposed the consistency property of an IMPR. They proposed two approaches to find the missing elements of incomplete IMPRs, i.e. "estimating step" and "adjusting step." To calculate the initial values of the missing element, a geometric mean method is used in the estimating step. To improve the initial values, another two different approaches are developed that is local optimizations model and iterative method. Jiang et al.(2015) method once getting the missing value, the consistency of IMPR will be checked by splitting the IMPRs into two MPRs. In this paper consistency of an arbitrary IMPRs are checked using the cyclic length of a directed graph. We have taken one example of Jiang et al. (2015) paper. Let us consider the example 2 of Jiang et al.(2015)

$$
A=\left[\begin{array}{cccc}
(1,1) & (3 / 2, *) & (1 / 3,1) & (1 / 2,1) \\
(*, 3 / 2) & (1,1) & (1 / 4,1 / 2) & (*, 2) \\
(1,1 / 3) & (2,1 / 4) & (1,1) & (1 / 3,1) \\
(1,1 / 2) & (2, *) & (1,1 / 3) & (1,1)
\end{array}\right]
$$


The order pair vertex matrix of IMPR $A$ is given

$$
V_{A}=\left[\begin{array}{cccc}
(0,0) & (1,0) & (0,0) & (0,0) \\
(0,0) & (0,0) & (0,0) & (0,1) \\
(1,0) & (1,0) & (0,0) & (0,0) \\
(1,0) & (0,0) & (1,0) & (0,0)
\end{array}\right]
$$

The lower and upper vertex matrix are

$$
V_{L}=\left[\begin{array}{llll}
0 & 1 & 0 & 0 \\
0 & 0 & 0 & 0 \\
1 & 1 & 0 & 0 \\
1 & 0 & 0 & 0
\end{array}\right], V_{U}=\left[\begin{array}{llll}
0 & 0 & 0 & 0 \\
0 & 0 & 0 & 1 \\
0 & 0 & 0 & 0 \\
0 & 0 & 0 & 0
\end{array}\right]
$$

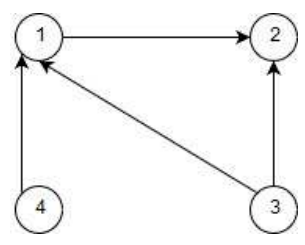

Fig. 14 Directed graph of $V_{L}$ of IMPR $A$

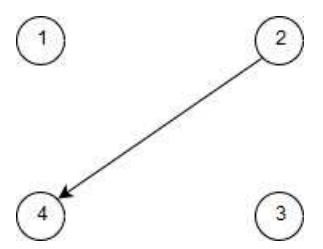

Fig. 15 Directed graph of $V_{U}$ of IMPR $A$

From figure 14 and 15, we conclude that no cycle is found in both the lower and upper vertex matrix. Therefore, the incomplete IMPR $A$ is consistent same as result of Jiang et al. (2015). Both the methods i.e our graphical methods and the Jiang et al. (2015) method are equivalent. But in graphical approach we can directly check the consistency of IMPR without finding the missing elements from the incomplete IMPR.

\section{Comparison with Zhang, Z. and Pedrycz,W.,(2017)}

Zhang, Z. and Pedrycz,W.,(2017), defined a new definition of consistency IMPR and proved that Xu's (Xu, Z. S., (2013)) definition of consistency and Jiang et al. (Jiang et al. (2015)) definition of consistency, are the special case of consistency defined by Zhang, Z. and Pedrycz,W.,(2017). According to Zhang, Z. and Pedrycz,W.,(2017) consistency is independent of alternative labels. For an multi-criteria decision making (MCDM) problem with $n$ decision alternatives, the DMs pairwise comparison information can be structured by differently labeling the $n$ - alternatives and yields $n$ ! IMPRs.

We take example 1 from the article by Zhang, Z. and Pedrycz, W.,(2017).

Consider three alternatives $A, B, C$ in an MCDM problems. A DM employs the pairwise comparison method to elicit his/her judgements. There are six possible cases for labeling the three alternatives. If the three alternatives $A, B, C$ are label by $x_{1}, x_{2}$ and $x_{3}$, then the DM's pairwise judgements are given by the following IMPR

$$
\begin{array}{rrr}
x_{1}: A & x_{2}: B & x_{3}: C \\
x_{1}: A \\
x_{2}: B \\
x_{3}: C
\end{array}\left(\begin{array}{ccc}
(1,1) & \left(\frac{2}{5}, \frac{3}{2}\right) & \left(\frac{2}{5}, \frac{3}{4}\right) \\
\left(\frac{3}{2}, \frac{2}{5}\right) & (1,1) & \left(1, \frac{1}{2}\right) \\
\left(\frac{3}{4}, \frac{2}{5}\right) & \left(\frac{1}{2}, 1\right) & (1,1)
\end{array}\right)
$$


By Jinag's (Jiang et al. (2015)) method it is consistent. According to our method, the order pair vertex matrix

$$
V=\left[\begin{array}{lll}
(0,0) & (0,1) & (0,0) \\
(1,0) & (0,0) & (0,0) \\
(1,1) & (0,1) & (0,0)
\end{array}\right]
$$

The lower and upper vertex matrix is

$$
V_{L}=\left[\begin{array}{lll}
0 & 0 & 0 \\
1 & 0 & 0 \\
1 & 0 & 0
\end{array}\right], \quad V_{U}=\left[\begin{array}{lll}
0 & 1 & 0 \\
0 & 0 & 0 \\
1 & 1 & 0
\end{array}\right]
$$
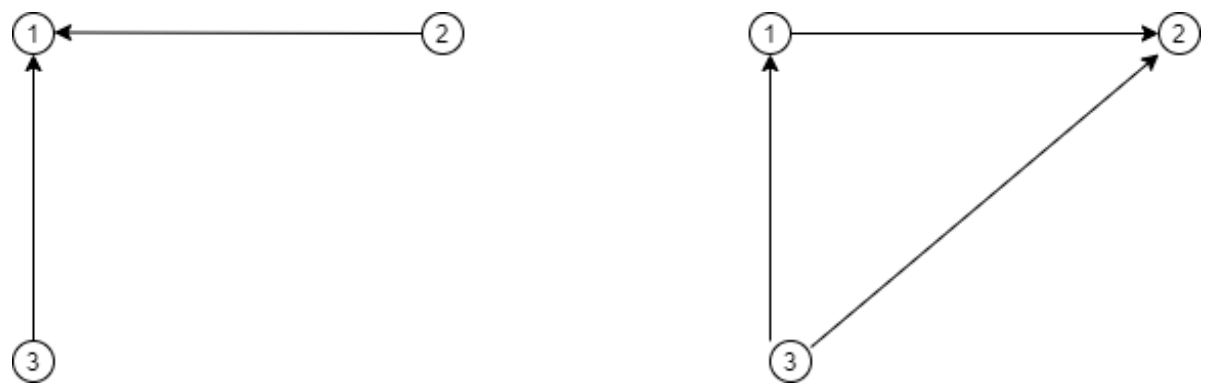

Fig. 16 Directed graph of $V_{L}$ and $V_{U}$ of IMPR of example 1 of Zhang, Z. and Pedrycz,W.,(2017)

From the directed graph 16, Both $V_{L}$ and $V_{U}$ are consistent because there are no circle was found. So, IMPR is consistent.

If the alternative $A, B, C$ are labeled by $x_{2}, x_{3}, x_{1}$, then the DM's pairwise judgements should be restructured by the following IMPR i.e.

$$
\begin{aligned}
& x_{1}: C \quad x_{2}: A \quad x_{3}: B \\
& x_{1}: C \quad(1,1) \quad\left(\frac{3}{4}, \frac{2}{5}\right) \quad\left(\frac{1}{2}, 1\right) \\
& x_{2}: A\left(\begin{array}{lll}
\left(\frac{2}{5}, \frac{3}{4}\right) & (1,1) \quad\left(\frac{2}{5}, \frac{3}{2}\right)
\end{array}\right) \\
& x_{3}: B\left(\begin{array}{lll}
\left(1, \frac{1}{2}\right) & \left(\frac{3}{2}, \frac{2}{5}\right) \quad(1,1)
\end{array}\right.
\end{aligned}
$$

This re-structured IMPR is inconsistent according to Jiang et al. (2015). But it is consistent, according to Zhang, Z. and Pedrycz, W.,(2017). Now same re-structured IMPR is consistent according to our method. The ordered pair vertex matrix is

$$
V=\left[\begin{array}{lll}
(0,0) & (0,0) & (0,0) \\
(1,1) & (0,0) & (0,1) \\
(1,0) & (1,0) & (0,0)
\end{array}\right]
$$

The lower and upper vertex matrix is

$$
V_{L}=\left[\begin{array}{lll}
0 & 0 & 0 \\
1 & 0 & 0 \\
1 & 1 & 0
\end{array}\right], \quad V_{U}=\left[\begin{array}{lll}
0 & 0 & 0 \\
1 & 0 & 1 \\
0 & 0 & 0
\end{array}\right]
$$

From the directed graph 17, Both $V_{L}$ and $V_{U}$ are consistent because there are no circle was found. So, re-structured IMPR is consistent. Therefore, the restructured IMPR, is consistent according to Zhang, Z. and Pedrycz,W.,(2017) as well as our method.

\section{Comparison with Jin et al. (2018)}



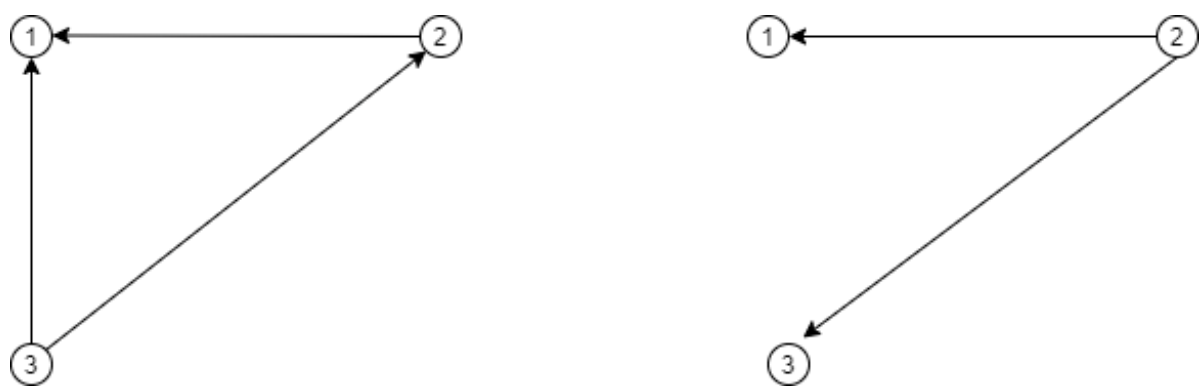

Fig. 17 Directed graph of $V_{L}$ and $V_{U}$ of re-structured IMPR of example 1 of Zhang, Z. and Pedrycz,W.,(2017)

Jin et al. (2018) proposed a method to derive normalized intuitionistic multiplicative weights on the basis of order consistent IMPR for decision making. Also, Jin et al. (2018) constructed an optimization model to generate the normalized intuitionistic multiplicative weights of IMPR, and compute the optimal deviation values to improve the consistency of the given IMPR, such that the repaired IMPR is constructed. To perform the comparison, we have taken the example 2 of (Jin et al. (2018)) the IMPR $P=\left(p_{i j}\right)$ i.e.

$$
P=\left(\begin{array}{cccc}
(1,1) & \left(\frac{5}{3}, \frac{1}{4}\right) & \left(7, \frac{1}{9}\right) & \left(3, \frac{1}{7}\right) \\
\left(\frac{1}{4}, \frac{5}{3}\right) & (1,1) & \left(\frac{5}{3}, \frac{1}{4}\right) & \left(\frac{5}{3}, \frac{1}{6}\right) \\
\left(\frac{1}{9}, 7\right) & \left(\frac{1}{4}, \frac{5}{3}\right) & (1,1) & \left(1, \frac{3}{5}\right) \\
\left(\frac{1}{7}, 3\right) & \left(\frac{1}{6}, \frac{5}{3}\right) & \left(\frac{3}{5}, 1\right) & (1,1)
\end{array}\right)
$$

According to Jin et al. (2018), the IMPR $P$ is non-order consistent. Then, they obtained the optimal normalized intuitionistic multiplicative weights, using goal programming model (M-2) of Jin et al. (2018). To construct the repaired consist IMPR, they utilize the optimal deviation values. Therefore, they get the repaired IMPR is consistent. Here we solve the same IMPR by using our method. The binary order pair vertex matrix of IMPR $P$ is

$$
V=\left(\begin{array}{cccc}
(0,0) & (1,0) & (1,0) & (1,0) \\
(0,1) & (0,0) & (1,0) & (1,0) \\
(0,1) & (0,1) & (0,0) & (0,0) \\
(0,1) & (0,1) & (0,1) & (0,0)
\end{array}\right)
$$

The lower and upper vertex matrices are,

$$
V_{L}=\left(\begin{array}{llll}
0 & 1 & 1 & 1 \\
0 & 0 & 1 & 1 \\
0 & 0 & 0 & 0 \\
0 & 0 & 0 & 0
\end{array}\right) ; \quad V_{U}=\left(\begin{array}{llll}
0 & 0 & 0 & 0 \\
1 & 0 & 0 & 0 \\
1 & 1 & 0 & 0 \\
1 & 1 & 1 & 0
\end{array}\right)
$$
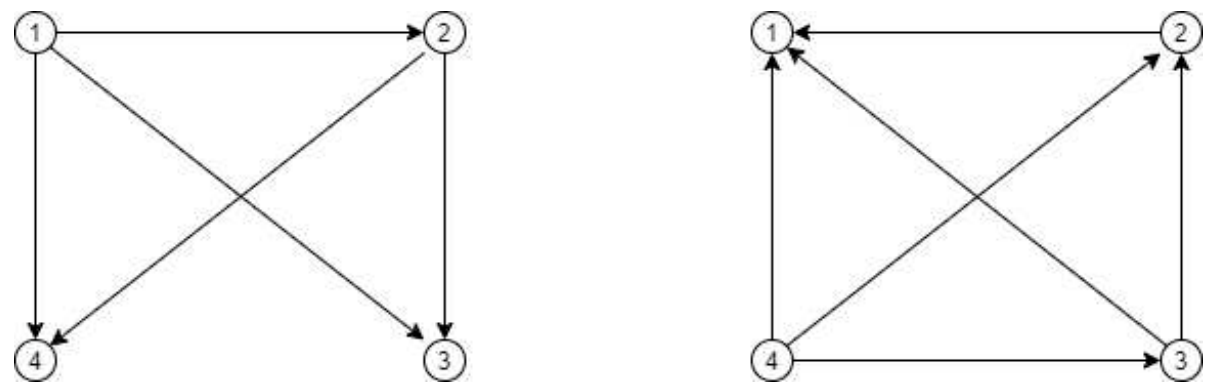

Fig. 18 Directed graph of $V_{L}$ and $V_{U}$ of IMPR of Jin et al. (2018)

From figure 18, it is clear that there is no cycle is found from the lower and upper vertex matrix. Both the $V_{L}$ and $V_{U}$ are consistent then the IMPR is also consistent. The IMPR $P$ is consistent by our 
method. Therefore both the methods gave the same result that proves the validity of our result and also in our method there is no need to solve any optimization problem.

\section{Conclusion}

In this paper, we have proposed two approaches to get the characterization of the consistency for intuitionistic multiplicative preference relation(IMPR). In the first approaches, we propose an algorithm to check the consistency of IMPR by using the cycles of various length in a directed graph and the same procedure apply for incomplete IMPRs also. The second approach proved isomorphic between the set of IMPRs and the set of asymmetric multiplicative preference relations. Also, consistency property is then checked of asymmetric preference relation using a directed graph that is used to get the consistency of IMPR.

Our future research will study the consistency of hesitant fuzzy preference relation HFPR using the cyclic length of a directed graph with the best additive consistency index, the worst additive consistency index and average additive consistency index of an HFPR (Zhang, Kou, Dong, 2018).

Zhang et al. (Zhang, Kou, Yu, Guo 2018) proposed an approach to deriving a priority weight vector from an incomplete HFPR using logarithmic least square method. Consistency index of an incomplete HFPR is defined. For an unacceptably consistent incomplete HFPR, an automatic algorithm is developed to improve the consistency. Also, we can check the consistency of incomplete HFPR using the cyclic approach that will result are then extended to propose a new procedure for the group analytic hierarchy process to deal with multi-criteria group decision-making problem.

Nowadays, linguistic large scale group decision-making problems are a handy tool in the decisionmaking process. Zhang et al. (2016) developed a new linguistic computational model with multi-granular linguistic distribution assessments for its application to large scale multi-attributes group decision-making problems with linguistic information. Our future research will study the consistency of IMPR with multigranular linguistic information based on the developed model. It will be more interesting to analyze the consistency of IMPR with multi-granular linguistic information using the cyclic length of the directed graph of IMPR. Also, our studies can be extended to the social network group decision-making framework (Ureña et al. 2019).

\section{Compliance with ethical standards}

Conflict of interest: The authors declare that they have no conflict of interest regarding the publication of this manuscript.

Ethical approval: This article does not contain any studies with human participants or animals performed by any of the authors.

\section{References}

Behret, H., (2014) Group decision making with intuitionistic fuzzy preference relations, Knowledge-Based Systems. 70, 33-43.

Capuano, N., Chiclana, F., Fujita, H., Herrera-Viedma, E., Loia, V., (2018) Fuzzy group decision making with incomplete information guided by social influence. IEEE Trans. On Fuzzy Systems 26(3) 1704-1718.

Chiclana, F., Herrera-Viedma, E., Alonso, S., Herrera. F., (2009) Cardinal Consistency of Reciprocal Preference Relations: A Characterization of Multiplicative Transitivity, IEEE Transactions on Fuzzy Systems 
17(1)14-23.

Chiclana, F., Herrera, F. „Herrera-Viedma, E., (2001) Integrating multiplicative preference relations in a multipurpose decision-making model based on fuzzy preference relations, Fuzzy Sets and Systems 122 277 Ü-291.

Ergu, D., Kou, G., Peng, Y., and Shi, Y.,(2011) A simple method to improve the consistency ratio of the pair-wise comparison matrix in ANP. Eur. J. Oper. Res., vol. 213, pp. 246Ü-259.

Fishburn, P. C., Utility Theory for Decision making. R.E. Krieger pub.Co.(1979).

Gong, Z.W., Li, F.J., Forrest, J., \& Zhao, Y., (2011) The optimal priority models of the intuitionistic fuzzy preference relation and application in selecting industries with higher meteorological sensitivity, Expert Systems with Applications. 38, 4394-4402.

Gong, Z. W., Li, L. S., Zhou, F.X., \& Yao, T. X., (2009) Goal programming approaches to obtain the priority vectors from the intuitionistic fuzzy preference relations, Computers \& Industrial Engineering. 57, 1187-1193.

Herrera-Viedma, E., Chiclana, F., Herrera, F., Alonso, S.,(2007) Group decision-making model with incomplete fuzzy preference relations based on additive consistency. IEEE Trans. Syst. Man Cybern. Ü Part B 37: 176Ü-189.

Herrera-Viedma, E., Herrera, F., Chiclana, F., and Luque, M.,(2004) Some issues on consistency of reciprocal relations, Eur. J. Oper. Res., vol. 154, 98Ü-109.

Jiang, Y., Xu, Z., Yu, X.,(2015) Group decision making based on incomplete intuitionistic multiplicative preference relations. Information Sciences 295: 33-52.

Jin, F., Ni, Z., Pei, L., Chen, H., Li, Y., (2018) Goal programming approach to derive intuitionistic multiplicative weights based on intuitionistic multiplicative preference relations. Int. J. Mach. Learn. \& Cyber. 9, 641Ü-650.

Li, C-C, Dong, Y., Xu, Y., Chiclana, F., Herrera-Viedma, E., Herrera, F., (2019) An overview on managing additive consistency of reciprocal preference relations for consistency-driven decision making and Fusion: Taxonomy and future directions. Information Fusion, 52, 143-156.

Nishizawa, K., (1995)A consistency improving method in binary AHP. Journal of Operations Research Society of Japan, vol. 38, No. 1, 21-33.

Nishizawa, K., (1996) A method to find elements of cycles in an incomplete directed graph and its Applications- Binary AHP and Petri Nets. Computers and Mathematics with Application. 33, 33-46.

Orlovsky, S. A, (1978) Decision making with a fuzzy preference relation. Fuzzy Sets Syst. 1: 155-167.

Ren, P., Xu, Z., Liao, H., (2016) Intuitionistic multiplicative analytic hierarchy process in group decision making. Computers \& Industrial Engineering, 101 513-524. 
Saaty, T. L, (1977) A scaling method for priorities in hierarchy structures. J. Math. Psychol. 15: 234-281.

Saaty, T. L, (1980) The Analytic Hierarchy process. McGraw-Hill, New York.

Ureña, M. R., Chiclana, F., Morente-Molinera, J. A., Herrera-Viedma E., (2015) Managing Incomplete Preference Relations in Decision Making: A Review and Future Trends. Information Sciences 302(1)14-32.

Ureña, R., Kou, G., Dong, Y., Chiclana, F., Herrera-Viedma E., (2019) A review on trust propagation and opinion dynamics in social networks and group decision making frameworks. Information Sciences, Volume 478, 461-475.

Xia, M. M., Xu, Z.S., Chen, J., (2013) Algorithms for improving consistency or consensus of reciprocal $[0,1]$-valued preference relations, Fuzzy Sets Syst. 216 108-133.

Xia, M.M., Xu, Z.S., Liao, H. C., (2013) Preference relations based on intuitionistic multiplicative information. IEEE Trans. Fuzzy Syst. 21, 113-Ü133.

Xu, Y., Herrera, F., (2019) Visualizing and rectifying different inconsistencies for fuzzy reciprocal preference relations, Fuzzy Sets and Systems, 362, 85-109.

Xu,Y., Herrera, F., Wang, H., (2016) A distance-based framework to deal with ordinal and additive inconsistencies for fuzzy reciprocal preference relations, Information Sciences, 328, 189-205.

Xu ,Y., Li,M., Cabrerizo ,F. J., Chiclana, F., and Herrera-Viedma, E., (2021) Algorithms to detect and rectify multiplicative and ordinal inconsistencies of fuzzy preference relations, IEEE Transactions on Systems, Man, and Cybernetics: Systems, 51(6), 3498-3511.

Xu,Y., Ma,F., Herrera, F.,(2019) Revisiting inconsistent judgments for incomplete fuzzy linguistic preference relations: Algorithms to identify and rectify ordinal inconsistencies, Knowledge-Based Systems, $163,305-319$.

Xu, Y., Patnayakuni, R., Wang, H. (2013) The ordinal consistency of a fuzzy preference relation, Information Sciences, 224, 152-164.

Xu, Y., Wang,Q., Cabrerizo,F. J., Herrera-Viedma, E., (2018) Methods to improve the ordinal and multiplicative consisetncy for reciprocal preference relation, Applied Soft Computing, 67, 479-493.

Xu, Z. S., (2007b) Intuitionistic preference relations and their application in group decision making. Information Sciences 177 (11) 2363-2379.

Xu, Z. S., (2013) Priority weight intervals derived from intuitionistic multiplicative preference relations. IEEE Trans. Fuzzy Syst. 21: 642-654.

Xu, Z., \& Liao, H., (2015) A survey of approaches to decision making with intuitionistic fuzzy preference relations, Knowledge-Based Systems, 80, 131-142. 
Xu, Z.S., Wei, C.P., (1999) A consistency improving method in the analytic hierarchy process, Eur. J. Oper. Res. 116, 443-449.

Zhang, Z. and Guo, C., (2017) Deriving priority weights from intuitionistic multiplicative preference relations under group decision-making settings, Journal of the Operational Research Society, 68, (12) $1582-1599$.

Zhang, Z., Guo, C., and Martnez, L., (2016) Managing Multigranular Linguistic Distribution Assessments in Large-Scale Multiattribute Group Decision Making, IEEE Transactions on Fuzzy Systems, Man, and Cybernetics: Systems, 47 (11), 3063-3076.

Zhang, Z., Kou, X., Dong, Q., (2018) Additive consistency analysis and improvement for hesitant fuzzy preference relations, Expert Systems With Applications, 98, 118-128.

Zhang, Z., Kou, X., Yu, W., Guo, C., (2018) On priority weights and consistency for incomplete hesitant fuzzy preference relations, Knowledge-Based Systems, 143, 115-126.

Zhang, Z. and Pedrycz, W.,(2018) Goal Programming Approaches to Managing Consistency and Consensus for Intuitionistic Multiplicative Preference Relations in Group Decision-Making, IEEE Transactions on Fuzzy Systems, DOI 10.1109/TFUZZ.2018.2818074.

Zhang, Z. and Pedrycz,W.,(2017) Models of Mathematical Programming for Intuitionistic Multiplicative Preference Relations, IEEE Transactions on Fuzzy Systems, 25 (4) 945-957. 